\title{
Synthesis and properties of graphene and its 2D inorganic analogues with potential applications
}

\author{
UTTAM GUPTA, K GOPALAKRISHNAN and C N R RAO* \\ Chemistry and Physics of Materials Unit, New Chemistry Unit, CSIR Centre of Excellence in Chemistry, International \\ Centre for Materials Science and Sheikh Saqr Laboratory, Jawaharlal Nehru Centre for Advanced Scientific Research, \\ Jakkur P.O., Bangalore 560064, India \\ *Author for correspondence (cnrrao@jncasr.ac.in)
}

MS received 21 March 2018; accepted 26 April 2018; published online 21 September 2018

\begin{abstract}
Discovery of the amazing properties of graphene has aroused great interest in other 2D materials. 2D inorganic analogues of graphene such as the transition metal dichalcogenides have been investigated widely and these materials, especially $\mathrm{MoS}_{2}$, exhibit many properties of interest. In particular, they possess properties of direct use in energy devices. In this article we review the synthesis and properties of the $2 \mathrm{D}$ materials of relevant transistors, sensors, photodetectors, supercapacitors and batteries as well as in oxygen reduction and hydrogen evolution reactions.
\end{abstract}

Keywords. 2D materials; energy applications; supercapacitors; Li batteries; oxygen reduction reaction; hydrogen evolution reaction.

\section{Introduction}

Graphene and transition metal dichalcogenides (TMDs) and such 2D layered materials exhibit fascinating properties, which can be employed for applications in transistors, sensing, energy storage and catalysis [1,2]. Graphene has exotic electronic properties, but due to its gapless nature, it cannot be used in certain electronic applications. There have been strategies to create a band gap in graphene by heteroatom doping or by surface modification. Nitrogen-doped graphene shows good electron mobility with n-type semiconducting behaviour, whereas boron-doped graphene is ambipolar [3]. Borocarbonitrides (BCNs) containing hexagonal $\mathrm{BN}$ and graphene domains of varying proportions have shown interesting electronic and gas adsorption properties [4]. TMDs include materials with a variety of properties like insulating $\mathrm{HfS}_{2}$, semiconducting $\mathrm{MoS}_{2}$ and metallic $\mathrm{TaS}_{2}$ and $\mathrm{NbSe}_{2}$. TMDs exhibit exotic features and with new properties arising from the valley polarization arising due to spin-orbit splitting [5]. In addition to the physiochemical properties, the layered materials have efficiently been used as materials in transistors, catalysts in water oxidation as well as reduction and oxygen reduction, supercapacitors and batteries [1]. It is noteworthy that cross-linked heterostructure of these 2D materials have shown interesting electrochemical and surface properties [6]. Stacking these 2D materials layer-by-layer increases not only the surface area but also the pore volume, which in turn enhances the gas sequestering properties. These newly designed cross-linked composites find applications in photochemical hydrogen evolution and supercapacitor applications. A schematic representation of the cross-linked 2D heterostructure is shown in scheme 1. In this article, we briefly describe the synthesis methods of the 2D materials and discuss the applications of graphene, TMDs and BCNs particularly in energy devices.

\section{Synthesis}

Synthesis of 2D nanosheets using micromechanical exfoliation of bulk single crystal by scotch tape gives highquality single-layer as well as few-layer samples, which are ideal for studying various condensed matter phenomena. However, the yields are poor and therefore to study their properties the liquid or thermal exfoliation methods are being used widely [7]. Novoselov et al [8] first demonstrated the micromechanical cleavage of graphite crystal by scotch tape method to synthesize single-layer graphene. Chemical vapour deposition (CVD) on different metal surfaces yields singlelayer to few-layer graphene with different carbon sources (methane, ethylene and acetylene) [9]. Subrahmanyam et al [10] produced good quantities of high-quality few-layer graphene (2-4 layers) using arc discharge of graphite in the presence of hydrogen atmosphere. Panchakarla et al [11] synthesized nitrogen-doped few-layer graphene by the arc-discharge method in the presence of ammonia or pyridine vapours to obtain 1-1.4 at\% of nitrogen content, whereas under CVD conditions Wei et al [12] obtained $\sim 9 \mathrm{wt} \%$. Li et al [13] thermally treated graphene oxide (GO) in the presence of ammonia at different temperatures $\left(300-1100^{\circ} \mathrm{C}\right)$ to obtain $5 \mathrm{wt} \%$ of nitrogen content. Boron-doped graphene synthesized under similar conditions by the arc-discharge method 

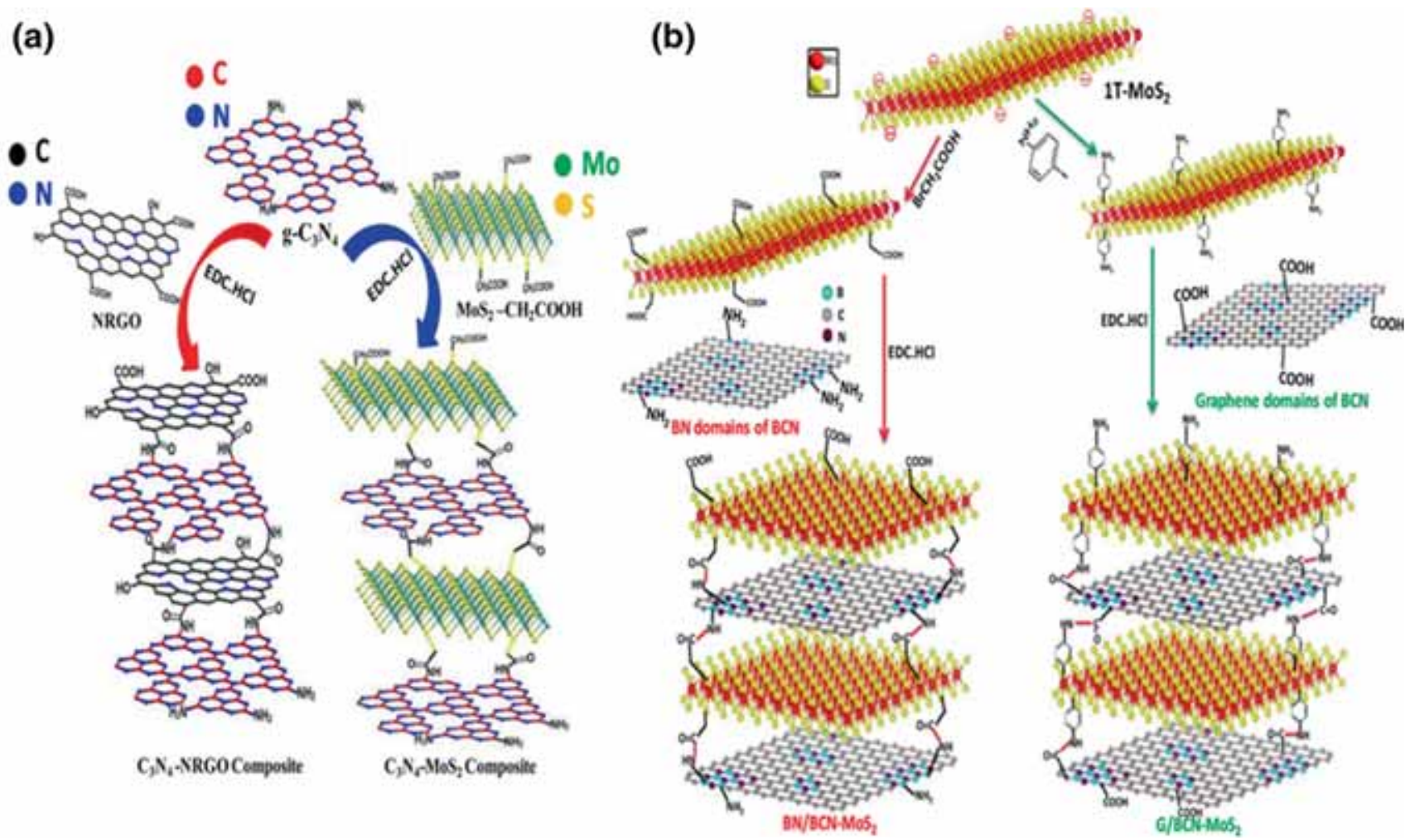

Scheme 1. Synthesis strategies for covalently bonded cross-linked (a) $\mathrm{C}_{3} \mathrm{~N}_{4}-\mathrm{NRGO}$ and $\mathrm{C}_{3} \mathrm{~N}_{4}-\mathrm{MoS} 2$ composites and (b) crosslinked $\mathrm{BN} / \mathrm{BCN}-\mathrm{MoS}_{2}$ and $\mathrm{G} / \mathrm{BCN}-\mathrm{MoS}_{2}$ nanocomposites (EDC = 1-ethyl-3-(3-dimethylaminopropyl)carbodiimide). Adapted from refs [6,26]. Copyright 2016 Royal Society of Chemistry. Copyright 2017 American Chemical Society.

yields 1-3 at $\%$ of boron content in the presence of diborane [11]. BCNs, which contain both graphene and boron nitride domains, can be synthesized by heating activated charcoal, urea and boric acid at $900^{\circ} \mathrm{C}$ in an inert atmosphere [14]. The composition of $\mathrm{BCN}$ can be tuned by varying the contents of urea and boric acid.

Direct sonication in solvents like N-methyl-2-pyrrolidone can exfoliate single or few layers of 2D TMDs like $\mathrm{MoS}_{2}$, $\mathrm{MoSe}_{2}, \mathrm{WS}_{2}$ and $\mathrm{TaS}_{2}$ (figure 1a) $[15,16]$. The dispersing medium should have surface energies comparable to those of the materials to overcome the cohesive energy between the layers [15]. Chemical exfoliation of the 3D-bulk crystal can be used for mass production $(\sim 100 \%)$ of exfoliated TMD nanosheets as exemplified by the use of n-butyl lithium dissolved in hexane as the intercalation agent (figure 1b) [17]. Interestingly, the semiconducting $2 \mathrm{H}$-form of $\mathrm{MoS}_{2}$ gets converted to the metallic 1T-MoS 2 after Li-intercalation and exfoliation [18], but the 1T-phase is not stable and reverts back to the $2 \mathrm{H}$-form.

Large area films of few-layer 2D TMDs have been grown by physical vapour deposition or CVD under suitable conditions. Vapour-phase transport recrystallization from powders, thermal decomposition of thio salts or vapour-phase deposition methods that include sulphurization or selenization of metals or metal oxides yield high-quality TMDs [19-22].
Physical and chemical properties and the compositions of the products are controlled by adjusting the ratio of precursors as well as reaction conditions (figure 1c) [19]. Various physicochemical properties of the solvents such as polarity, viscosity and softness can affect the solubility and transport behaviour of the precursors during the synthesis process [23]. Mahler et al [24] have synthesized 1T- $\mathrm{WS}_{2}$ and $2 \mathrm{H}-\mathrm{WS}_{2}$ nanosheets in a mixture of oleylamine, $\mathrm{WCl}_{6}$, oleic acid and $\mathrm{CS}_{2}$ by solvothermal method (figure 1d). The most important aspect of the solvothermal method is that it prevents the TMDs from oxidizing. Thus, the moderate temperatures together with the advantage of fast reaction kinetics and short processing time render it as a promising route to prepare homogeneous products on a large scale.

Cross-linked heterostructures (BCN-MoS 2 [25], $\mathrm{MoS}_{2}-$ nitrogen-doped graphene [26]) can be synthesized using a coupling reagent like $\mathrm{N}$-(3-(dimethylamino)propyl) $\mathrm{N}^{\prime}$ ethylcarbodiimide hydrochloride (EDC. $\mathrm{HCl}$ ) (scheme 1). With this strategy, any two layered structures can be crosslinked with suitable reagents [6]. $\mathrm{C}_{3} \mathrm{~N}_{4}$ can be linked to both $\mathrm{MoS}_{2}$ and graphene by EDC coupling. BCNs and functionalized graphene can be linked to $\mathrm{MoS}_{2}$ and other layered structures. The cross-linking strategy offers versatility in linking two different layered materials, providing layer-by-layer stacking and new properties. 

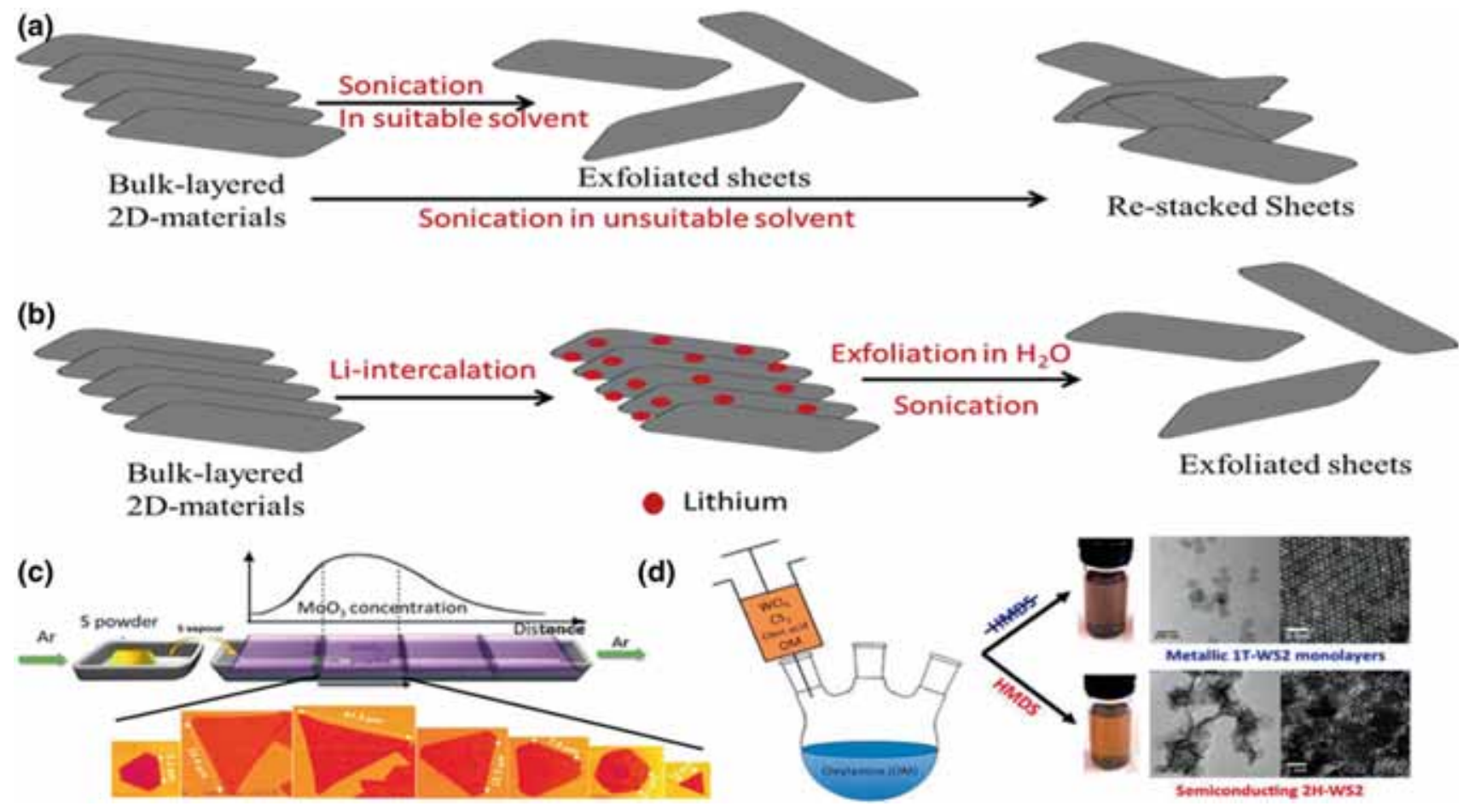

Figure 1. (a) Exfoliation of bulk 2D-layered materials either by probe or bath sonication in a suitable solvent can give high-quality single- to few-layer sheets. In unsuitable solvents the exfoliated sheets can be restacked. (b) Chemical exfoliation by Li-intercalation followed by exfoliation in water gives large-scale exfoliated single- to few-layer sheets. (c) Large-area sheets of 2D TMDs under suitable conditions can be synthesized. A schematic of synthesis of $\mathrm{MoS}_{2}$ nanoflakes with different shapes. The concentration of $\mathrm{MoS}_{2}$ is the determining factor in the evolution of these shapes during synthesis. The Ar flow rate is $100 \mathrm{sccm}$ and reaction occurs on $300 \mathrm{~nm} \mathrm{SiO}_{2}$ coated Si-chip. Adapted with permission from ref. [19]. (d) Schematic of synthesis of $1 \mathrm{~T}$ and $2 \mathrm{H}-\mathrm{WS}_{2}$. The crystal structure is different when HDMS is added, which shows the determining effect of the solvent. With suitable reaction conditions we can synthesize 2D materials from their precursors. Adapted from ref. [24]. Copyright 2014 American Chemical Society.

\section{Transistors}

Graphene is the most widely studied 2D material for transistor properties because of its extraordinary mobility of $\sim 25000 \mathrm{~cm}^{2} \mathrm{~V}^{-1} \mathrm{~s}^{-1}$ at room temperature [27]. However, it is not possible to switch off the FET because of its delocalized electrons, hence showing poor on-off ratios $(<10)$. Graphene nanoribbons with a small band gap (140 meV) show a conventional band transport at room temperature [28]. Optimization of graphene nanoribbons can give rise to useful FET properties. Reduced graphene oxide (RGO) has been used as a transistor, but the performance is less as compared with mechanically exfoliated graphene [29]. Fiori et al [30] have simulated carbon-dependent transfer characteristics in BCNs. The carbon-rich BCN showed high current transport, but poor on-off ratio, whereas the $\mathrm{BN}$-rich $\mathrm{BCN}$ showed high on-off ratios. BCNs have mobility ranges from 6.4 to $10 \mathrm{~cm}^{2} \mathrm{~V}^{-1} \mathrm{~s}^{-1}$ in FETs for carbon-rich samples [31]. Other 2D materials like TMDs, silicene and phosphorene have been recently studied [32]. The electronic mobility shows a relationship with the band gap and is shown for graphene, TMDs and other 2D materials and 3D materials in figure 2a [33].
TMDs possess diverse electronic properties from metal to insulator, with the band gap (1.1-2.2 eV) increasing with a decrease in the number of layers [34]. FETs based on mechanically exfoliated $\mathrm{MoS}_{2}$ of thickness 8-40 nm with back-gated FET configuration have shown mobilities in the range of $0.1-10 \mathrm{~cm}^{2} \mathrm{~V}^{-1} \mathrm{~s}^{-1}[8,35] . \mathrm{MoS}_{2}$ has gained attention over other TMDs because it is a robust, naturally abundant, highquality crystal, which is readily mechanically exfoliated. Late et al [36] observed controllable hysteretic behaviour in atomically thin $\mathrm{MoS}_{2}$ FET induced by adsorbed water molecules on $\mathrm{MoS}_{2}$ surface and it can find potential applications in nonvolatile memory devices. The first top-gated transistor based on monolayer $\mathrm{MoS}_{2}$ reported by Radisavljevic [37] exhibited an on-off ratio of $10^{8}$ with n-type conductivity and mobility of $>200 \mathrm{~cm}^{2} \mathrm{~V}^{-1} \mathrm{~s}^{-1}$ at room temperature (figure $2 \mathrm{~b}$ ). The high- $k$ dielectric $\mathrm{HfO}_{2}$ benefits the mobility of monolayer of $\mathrm{MoS}_{2}$ [38-40], while the top gate geometry can allow a reduction in the voltage necessary to switch the device, which allows integration of multiple devices on a single substrate. Theoretically, a top-gated $\mathrm{MoS}_{2}$ transistor with gate lengths of $15 \mathrm{~nm}$ can operate in a ballistic regime with on-current as

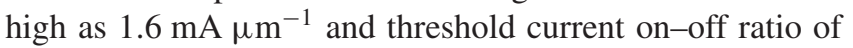


(a)

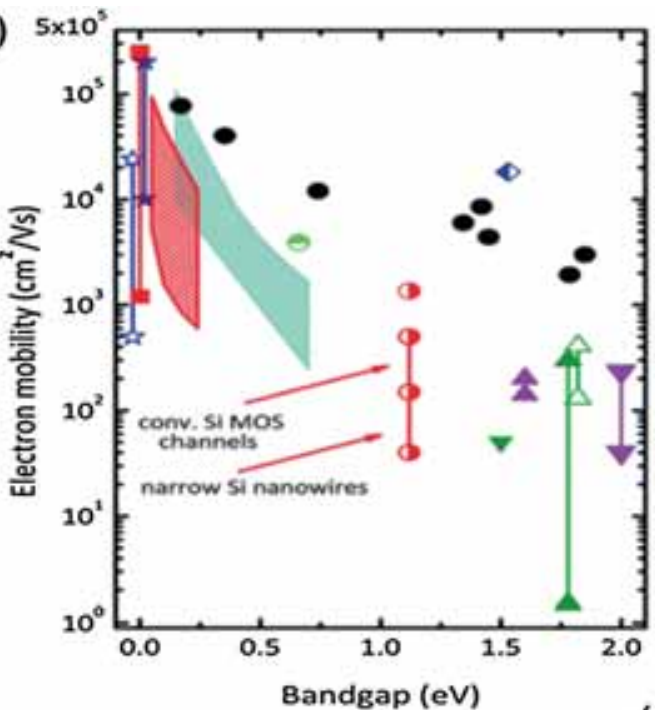

(b)

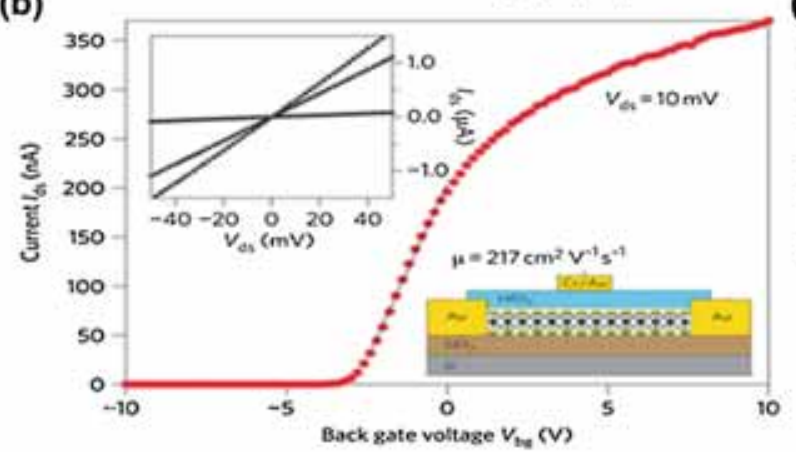

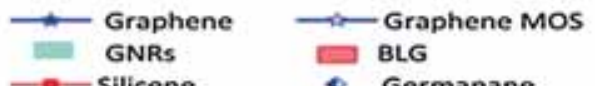

- Silicene Germanane

$\infty \operatorname{Mos}_{2}$ (theory) $\longrightarrow \operatorname{Mos}_{2}$ (experiment)

v Mose (experiment)

$-\mathrm{WS}_{2}$ (experiment)

WSe, (experiment)

a Si (bulk)

s Ge (bulk)

- III-Vs (bulk)

(c)

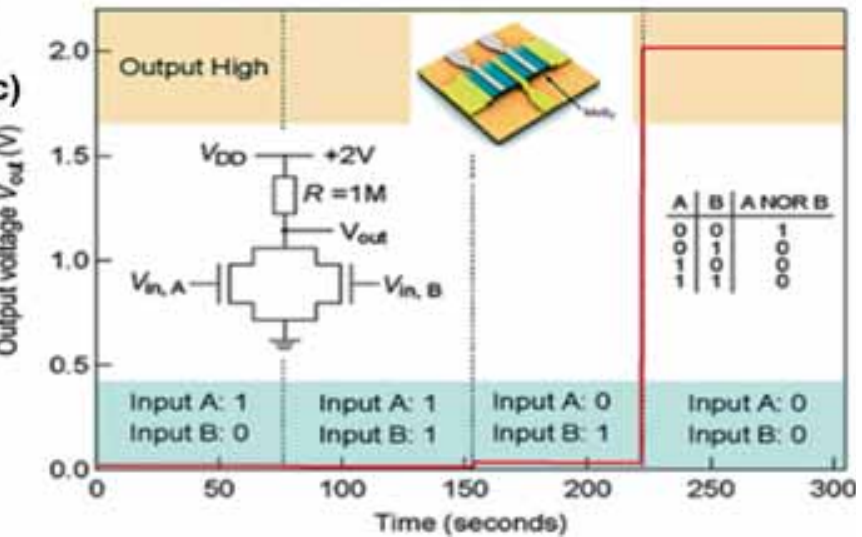

Figure 2. (a) Comparison of room-temperature electron mobility $v s$. band gap for different materials with 2D materials like graphene and TMDs. The figure was adapted with permission from ref. [33]. (b) Schematic of cross-sectional view of the top-gate FET of a monolayer $\mathrm{MoS}_{2}$ (inset in right hand corner). Transfer characteristic for the FET with applied bias voltage $V_{\mathrm{ds}}(10 \mathrm{mV})$. The back-gate voltage $\left(V_{\mathrm{bg}}\right)$ is applied to the substrate while the top gate is disconnected. Drain-source current $\left(I_{\mathrm{ds}}\right)$-drain-source voltage $\left(V_{\mathrm{ds}}\right)$ curves acquired for $V_{\mathrm{bg}}$ at 0,1 and $5 \mathrm{~V}$. The figure is adapted from ref. [37]. (c) Schematic of a device for NOR gate based on single-layer MoS 2 (inset middle top). Two monolayer $\mathrm{MoS}_{2}$ transistors are connected in parallel using an external $1 \mathrm{M} \Omega$ resistor as a load (left inset) the logic circuit is formed. The output voltage $\left(V_{\text {out }}\right)$ is shown in various regimes for four different sets of input states $(1,0),(1,1),(0,1)$ and $(0,0)$ with response only, with both inputs in the low state. The truth table shows that these combinations can be shown as NOR gate operations (inset). Adapted from ref. [42]. Copyright 2016 Elsevier. Copyright 2011 Springer Nature. Copyright 2011 American Chemical Society.

$10^{10}$ [41]. Radisavljevic et al [42] built a functional electronic circuit based on $\mathrm{MoS}_{2}$ that is capable of performing digital logic operations (figure 2c). Ambipolar transport demonstrated in a thin (10 $\mathrm{nm}$ thick) $\mathrm{MoS}_{2}$ electric double layer transistor using an ionic liquid as the gate to reach extremely high carrier concentrations of $10^{14} \mathrm{~cm}^{2}$ with on-off ratio of 200 [43].

A promising approach is to stack different 2D-layered materials to make vertical heterostructures and hybrid devices with operating principles that are unlike those of conventional devices. Recently, FET with high switching ratio was achieved using two independently controlled graphene layers are separated by thin $\mathrm{MoS}_{2}$ or h-BN layers, which act as tunneling barriers [44]. Similar heterojunctions with $\mathrm{MoS}_{2}$ and highly doped germanium have been reported to exhibit low currents at a supply voltage $<100 \mathrm{mV}$ [45]. The implantation of $2 \mathrm{D}$ materials in electronic devices is still at research level, and some basic techniques are under development. The challenge is to increase the current density and reduce the contact resistance at least on the order of $<100 \Omega$, especially for short channel length. A heterostructure design with doping or controlled growth can result in thinnest possible FETs with several opportunities in 2Dcrystal electronics.

\section{Sensors}

\subsection{Gas sensors}

2D-layered materials, like graphene, are promising sensing materials due to their high surface area and low-single to noise ratio compared with $1 \mathrm{D}$ or $0 \mathrm{D}$ structures $[46,47]$. Graphene has substantial conductivity and exhibits changes 

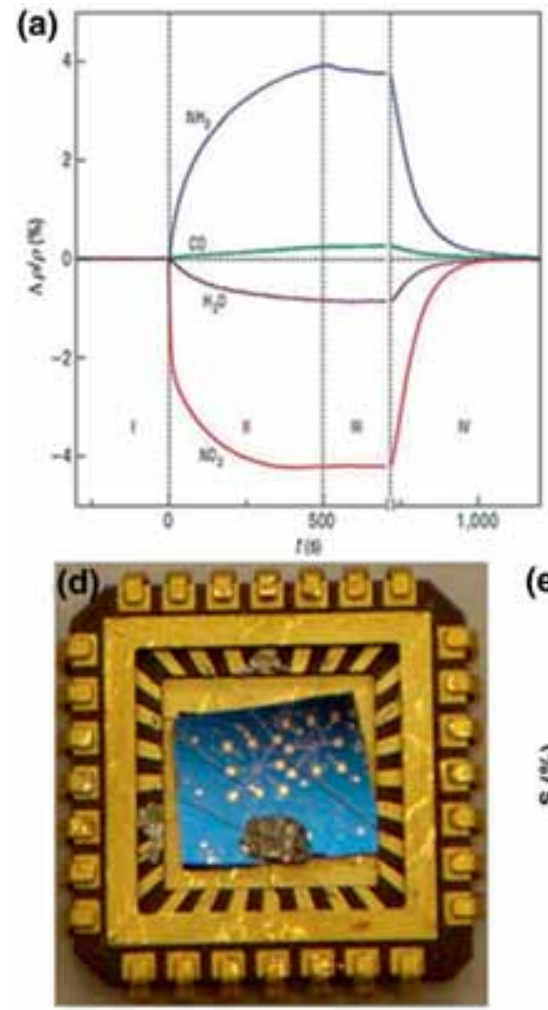

(b)

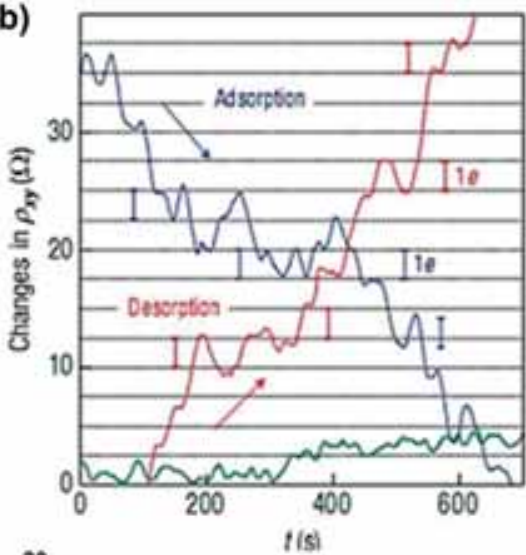

(e)

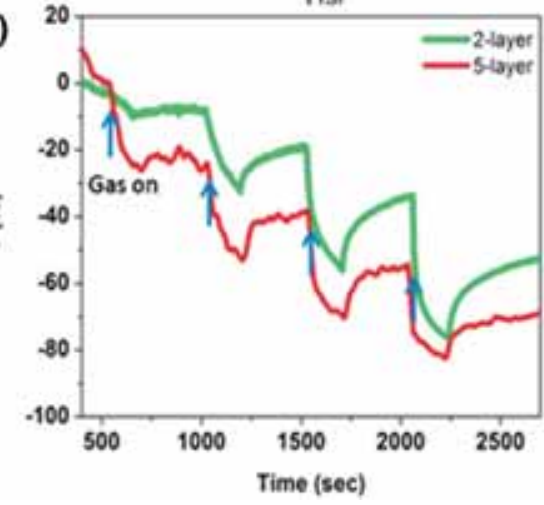

(c)

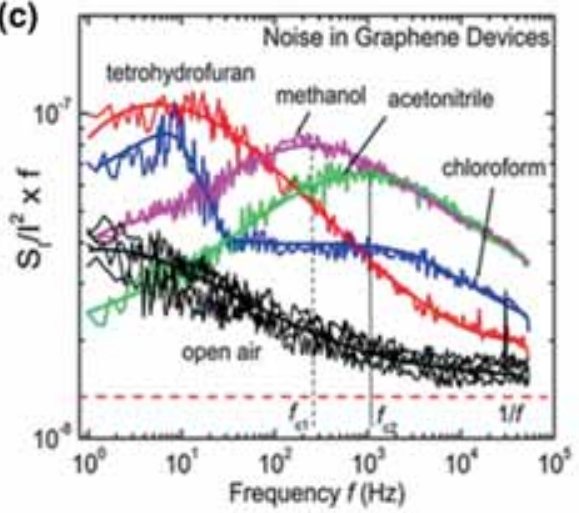

(f)

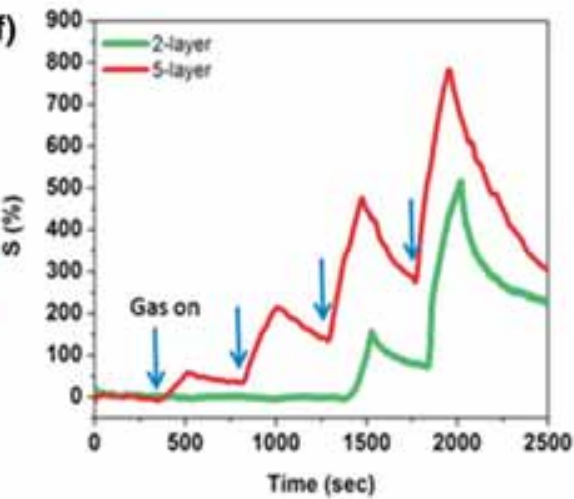

Figure 3. (a) The graph represents change in resistivity with respect to time over different stages of gas sensing procedure of various gases (1 ppm). Vacuum (Region I), when gas in introduced (Region II) on exposure to gas, evacuation of the experimental set-up (Region III) and annealing at $150^{\circ} \mathrm{C}$ to de-adsorb gas (Region IV). The positive sign indicates electron doping while the negative sign indicates hole doping. (b) The change of Hall resistivity $\rho_{x y}$ observed near the neutrality point through adsorption (blue curve) and desorption (red curve) of diluted $\mathrm{NO}_{2}$. Green curve is when the sensor is exposed to pure He, which is used as a reference. Adapted from ref. [49]. (c) Analysis of product of noise spectral density $\left(\mathrm{SI} / \mathrm{I}_{2}\right)$ multiplied by frequency $(f) v s$. $f$ for the sensor upon exposure to different vapour samples along with open air. Adapted from ref. [52]. (d) Photograph of the transistor-based $\mathrm{MoS}_{2}$-sensing device mounted on the chip. Sensing behaviour of (e) $\mathrm{NH}_{3}$ and (f) $\mathrm{NO}_{2}$ gas with and without applying back-gate voltage $\left(V_{\mathrm{g}}=15 \mathrm{~V}\right)$ for two-layer MoS 2 (arrows indicate concentration of gases at 100, 200, 500 and 1000 ppm of each gas). Adapted from ref. [64]. Copyright 2017 Springer Nature. Copyright 2017 American Chemical Society.

in conductivity on interaction with foreign molecules. For instance, $\mathrm{H}_{2} \mathrm{O}$ molecule does not induce much change in the localized impurity states in graphene but alters the conductivity noticeably (figure 3a) [48]. An electron-donating molecule such as $\mathrm{NH}_{3}$ decreases the concentration of carriers while $\mathrm{NO}_{2}$, an electron-withdrawing molecule, increases the concentration of carriers (figure 3a). Similarly, halogen molecules or alkali elements act as dopants and contribute electron or hole doping. A single-layer graphene-based gas sensor synthesized by Schedin et al [49] has a detection limit of ppb for $\mathrm{NO}_{2}$ molecule, which was further improved using Hall geometry (figure $3 b$ ). Since then, single-layer graphene has been an attractive material to detect gases or small organic molecules like methanol, THF, acetonitrile and chloroform [50,51]. Interestingly, vapours of different molecules induce noise with a different frequency, which distinguishes the signal to detect them both qualitatively and quantitatively (figure 3c) [52]. A drawback of pristine graphene is that it is highly sensitive to contaminants which can be overcome with illumination with UV light as exhibited by Chen et al [53]. The sensor was highly sensitive to $\mathrm{N}_{2} \mathrm{O}, \mathrm{NH}_{3}, \mathrm{SO}_{2}$ and $\mathrm{H}_{2} \mathrm{O}$ under flowing $\mathrm{N}_{2}$ carrier gas with detection limits in the range of 39-136 ppt.

Chemically exfoliated graphene, hydrogenated graphene and RGO show excellent sensing properties [47]. RGO has shown high sensitivity to $\mathrm{H}_{2} \mathrm{O}$ and gases like $\mathrm{NO}_{2}$ and $\mathrm{Cl}_{2}$ at low concentrations [54,55], which was further increased by sulphonation [56]. Chemically reduced few-layer graphene efficiently detects DNA bases, nicotinamide adenine dinucleotide (NADH), neurotransmitters, oxidase/dehydrogenase-related molecules, other molecules like acetaminophen, uric acid or ascorbic acid and other biomolecules [57,58]. Electrochemical sensors based on RGO can detect biomolecules at minute concentrations through impedance spectroscopy $[59,60]$. BCN was earlier used as a sensor for real-time monitoring of slag oxidation by 
measuring the partial pressure of oxygen [61]. Recently, BCN has shown sensor properties for uric acid and dopamine [62].

Like graphene, $\mathrm{MoS}_{2}$ has shown remarkable sensors properties. FET devices of mechanically exfoliated $\mathrm{MoS}_{2}$ have been used as NO sensors with a sensitivity of 300$800 \mathrm{ppb}$, depending on the number of layers [63]. Similar to graphene, acceptor molecule like $\mathrm{NO}_{2}$ and electron donating molecule like $\mathrm{NH}_{3}$ increases or decreases the resistance of $\mathrm{MoS}_{2}$, respectively, based on charge transfer mechanism (figure 3d-f) [64]. Single-layer $\mathrm{MoS}_{2}$ can detect DNA and other biomolecules by photoluminescence spectroscopy [65]. $\mathrm{MoS}_{2}$ can distinguish between the single- or double-stranded DNA due to the difference in fluorescence-quenching ability [66]. DNA can also be detected with FET devices based on $\mathrm{MoS}_{2}$ [67]. Graphene has generally better sensing ability. The sensing properties of the gases by graphene and other TMDs are listed in table 1.

\subsection{Photodetectors}

Single-layer graphene has optical absorption over a wide range as well as strong interband transitions and acts as an ultrafast photodetector [70,71]. Chemically reduced graphene offers cheaper, tuneable, scalable and solution-processed UV and IR detectors with good photoresponse and on-off ratio $[72,73]$. The detectors show a strong response to signals as weak as human-hand. However, for photodetector and photovoltaic applications, materials with suitable band gap is preferred.

$\mathrm{MoS}_{2}$ possesses band gap with properties suitable for photodetector and photovoltaic applications. Single- and double-layer $\mathrm{MoS}_{2}$ have been used as a detector of green light, while three-layer $\mathrm{MoS}_{2}$ detects red light [74]. Chemically exfoliated $\mathrm{MoS}_{2}$ was reported to show near-IR absorbance, where $\mathrm{MoS}_{2}$ shows 7.8 times greater absorbance than that of RGO [75,76]. Phototransistors made using single-layer $\mathrm{MoS}_{2}$ have shown fast response with a switching duration of $50 \mathrm{~ms}$ and good photosensitivity [77]. The responsivity of such devices can be tailored by the gate voltage, making them even better than graphene-based devices. Being a direct-bandgap semiconductor, single-layer $\mathrm{MoS}_{2}$ phototransistors, with improved contacts and positioning techniques, show $\sim 106$ times better responsivity than that of graphene [78]. Heterostructures of TMDs have shown strong sensing properties for both light and matter [79]. Photodetectivity of graphene and TMD-based devices is shown in table 2 .

\section{Supercapacitors}

Supercapacitors are electrochemical energy storing devices that find applications because of their high energy and power density [86]. Carbon-based materials such as carbon nanotubes, carbon nanofibres and graphene have shown excellent supercapacitor properties due to their high surface area and electrical conductivity [87]. Vivekchand et al [88] reported the first graphene-based supercapacitor, wherein few-layered graphene exhibits high specific capacitance up to $117 \mathrm{~F} \mathrm{~g}^{-1}$ in aqueous electrolytes and an energy density of $31.9 \mathrm{Wh} \mathrm{kg}^{-1}$ in an ionic liquid. Supercapacitors made of chemically modified graphene have shown a maximum specific capacitance of $135 \mathrm{~F} \mathrm{~g}^{-1}$ in aqueous electrolyte [89], while supercapacitors made of activated graphene with macro- and mesopores have shown a capacitance of $174 \mathrm{~F} \mathrm{~g} \mathrm{~g}^{-1}$ in an organic electrolyte with an energy density and power density of $74 \mathrm{Wh} \mathrm{kg}^{-1}$ and $338 \mathrm{~kW} \mathrm{~kg}^{-1}$, respectively [90]. The surface area of graphene has been increased up to $2400 \mathrm{~m}^{2} \mathrm{~g}^{-1}$ by microwave exfoliation or by $\mathrm{KOH}$ activation of GO. Such

Table 1. The sensing properties of graphene and TMDs.

\begin{tabular}{|c|c|c|c|}
\hline Sensor material & Target gas & Sensitivity and limit of detection & References \\
\hline \multicolumn{4}{|l|}{ Graphene } \\
\hline $\begin{array}{l}\text { Mechanically exfoliated } \\
\text { graphene }\end{array}$ & $\mathrm{NO}_{2}$ & $\begin{array}{c}\Delta R>2.5 \Omega \text { for one electron; } \\
\text { single-molecule detection }\end{array}$ & [49] \\
\hline $\begin{array}{l}\text { Mechanically exfoliated } \\
\text { graphene }\end{array}$ & $\begin{array}{l}\mathrm{CH}_{3} \mathrm{OH}, \mathrm{C}_{2} \mathrm{H}_{5} \mathrm{OH}, \mathrm{THF}, \\
\mathrm{CH}_{3} \mathrm{CN}\end{array}$ & $\begin{array}{l}\text { Using frequency } v s \text {. resistivity } \\
\text { pattern variation of each vapour } \\
\text { molecule }\end{array}$ & {$[52]$} \\
\hline CVD-grown graphene & $\mathrm{NO}_{2}, \mathrm{O}_{2}, \mathrm{SO}_{2}, \mathrm{NO}$ & $\begin{array}{l}\Delta R=0.2-0.5 \% \mathrm{ppm}^{-1} \\
158 \mathrm{ppq}-103 \mathrm{ppt}\end{array}$ & {$[53]$} \\
\hline RGO (printable sensor) & $\mathrm{NO}_{2}, \mathrm{Cl}_{2}$ & $500 \mathrm{ppb}-100 \mathrm{ppm}$ & {$[68]$} \\
\hline $\begin{array}{l}\text { Sulphonated/ethylenediamine- } \\
\text { modified RGO }\end{array}$ & $\mathrm{NO}_{2}$ & $\begin{array}{l}\Delta G / G=0.443 \mathrm{ppm}^{-1} \\
3.6 \mathrm{ppm}\end{array}$ & {$[56]$} \\
\hline \multicolumn{4}{|l|}{$\mathrm{MoS}_{2}$} \\
\hline $\begin{array}{l}\text { Mechanically exfoliated } \\
1 \mathrm{~L}-\mathrm{MoS}_{2}\end{array}$ & $\begin{array}{l}\text { Selective detection of electron donors } \\
\text { like triethylamine, selectivity better } \\
\text { than that of CNT }\end{array}$ & $10 \mathrm{ppb}$ & [69] \\
\hline $\begin{array}{l}\text { Mechanically exfoliated } \\
2 \& 5 \mathrm{~L}-\mathrm{MoS}_{2}\end{array}$ & $\mathrm{NO}_{2}, \mathrm{NH}_{3}$, relative humidity & $1.37 \% \mathrm{ppm}^{-1}$ & {$[64]$} \\
\hline
\end{tabular}


Table 2. Photo-detection activities of graphene and 2D-TMDs.

\begin{tabular}{lccc}
\hline Device type & Responsivity & Quantum efficiency (\%) & References \\
\hline $\begin{array}{l}\text { Graphene } \\
\text { Photoconduction }\end{array}$ & $\sim 0.1 \mathrm{~mA} \mathrm{~W}^{-1}$ & - & \\
Heterojunction & $100 \mathrm{~mA} \mathrm{~W}^{-1}$ & - & {$[80]$} \\
Bolometric & $0.2 \mathrm{~mA} \mathrm{~W}^{-1}$ & $10-30 \%$ & {$[81]$} \\
TMDs & & & {$[82]$} \\
$\begin{array}{l}\text { In-plane } \\
\text { Photoconduction }\end{array}$ & $880 \mathrm{~A} \mathrm{~W}^{-1}$ & - & {$[78]$} \\
$\quad \begin{array}{l}\text { Heterojunction } \\
\text { Out-of-plane }\end{array}$ & $210 \mathrm{~mA} \mathrm{~W}^{-1}$ & $\sim 30 \%$ & {$[83]$} \\
Photoconduction & $100 \mathrm{~mA} \mathrm{~W}^{-1}$ & $0.2 \%$ & {$[84]$} \\
Heterojunction & $\sim 100 \mathrm{~mA} \mathrm{~W}^{-1}$ & $\sim 10-30 \%$ & {$[85]$} \\
\hline
\end{tabular}

high-surface-area graphene shows a specific capacitance of $166 \mathrm{~F} \mathrm{~g}^{-1}$ [91] and $191 \mathrm{~F} \mathrm{~g}^{-1}$ [92]. In-plane fabricated fewlayered RGO supercapacitor electrode shows a capacitance of $394 \mu \mathrm{F} \mathrm{cm}^{-1}$ in PVA- $\mathrm{H}_{3} \mathrm{PO}_{4}$ solid-state electrolyte [93]. The performance of graphene clearly depends upon the number of layers, surface area and pore structure $[88,91]$. The intrinsic capacitance of graphene can be increased by doping with hetero-atoms such as nitrogen and boron, which in turn tune the electronic properties [3].

Nitrogen-plasma-processed graphene shows nearly four times higher capacitance $\left(\sim 280 \mathrm{~F} \mathrm{~g} \mathrm{~g}^{-1}\right)$ than that of unprocessed graphene with excellent cycling stability and power densities [94]. The study predicts that the nitrogen atoms present between edges and basal planes of graphene are the primary reason behind the increase in capacitance. Crumbled nitrogen-doped graphene synthesized by heating $\left(750\right.$ or $900^{\circ} \mathrm{C}$ ) GO in ammonia shows high capacitance $\left(248 \mathrm{~F} \mathrm{~g}^{-1}\right)$ due to its high pore volume, nitrogen content and the wrinkles formed during the synthesis [95]. RGO treated with urea at different temperatures shows increase in specific capacitance due to the increase in nitrogen content [96]. Heavily nitrogenated graphene (NGO) synthesized by microwave method using urea as the nitrogen source shows extraordinary supercapacitor properties [97]. Figure 4a shows the cyclic voltammogram of heavily nitrogenized graphene. The cyclic voltammogram is quasi-rectangular, which confirms it to be an ideal capacitor with a specific capacitance of $461 \mathrm{~F} \mathrm{~g}^{-1}$. The Nyquist plot shows little charge transfer resistance in these materials as shown in figure $4 \mathrm{~b}$. The stability of NGO is shown in figure $4 \mathrm{~b}$, where the material shows excellent cyclic stability with just $2.7 \%$ decrease from the initial specific capacitance as shown in figure $4 \mathrm{c}$. The energy density and power density are $44.4 \mathrm{Wh} \mathrm{kg}^{-1}$ and $852 \mathrm{~W} \mathrm{~kg}^{-1}$, respectively (figure $4 \mathrm{~d}$ ). The reason behind the increase in specific capacitance is the high nitrogen content of graphene. There are three different nitrogens present in the $\mathrm{N}$-doped graphene, namely pyrrolic, pyridinic and graphitic nitrogen. Pyrrolic and pyridinic nitrogens are found to be electrochemically active and cause an increase in capacitance, whereas the graphitic nitrogen increases the conductivity of the material [98].

Boron-doped graphene also shows an increase in capacitance compared with pristine graphene. Boron-doped graphene synthesized by heating $\mathrm{GO}$ with boric oxide shows a capacitance of $172 \mathrm{~F} \mathrm{~g}^{-1}$ at $0.5 \mathrm{~A} \mathrm{~g}^{-1}$ with good stability over extended cycling [99]. The increase in capacitance is due to the formation of oxygen-containing functional groups such as $\mathrm{BC}_{2} \mathrm{O} / \mathrm{BCO}_{2}$ while doping. Porous $\mathrm{B}$-doped graphene made by Fried ice method exhibits a high surface area of $622 \mathrm{~m}^{2} \mathrm{~g}^{-1}$ and a capacitance of around $281 \mathrm{~F} \mathrm{~g}^{-1}$ [100]. Borane-reduced GO shows a capacitance of $200 \mathrm{~F} \mathrm{~g}^{-1}$ with good stability over 4000 cycles [101]. Laser-patterned B-doped graphene micro-supercapacitor shows an excellent areal capacitance of $16.5 \mathrm{mF} \mathrm{cm}^{-2}$, which is 3 times higher than that of pristine graphene devices [102]. Dielectric barrier discharge plasma-based boron-doped graphene exhibits a capacitance of $446 \mathrm{~F} \mathrm{~g}^{-1}$ at $0.5 \mathrm{~A} \mathrm{~g}^{-1}$ [103]. Sponge-like B-doped graphene nanosheets synthesized hydrothermally using boric acid shows a capacitance of $113 \mathrm{~F} \mathrm{~g}^{-1}$ at $1 \mathrm{~A} \mathrm{~g}^{-1}$ [104].

BCN synthesized by heating urea and boric acid with activated charcoal shows excellent supercapacitor properties [96]. In addition to capacitor properties, it also possesses high surface area and gas sequestering abilities [14]. $\mathrm{BC}_{4.5} \mathrm{~N}$ has the highest specific capacitance of 178 and $240 \mathrm{~F} \mathrm{~g}^{-1}$ in an aqueous electrolyte and in an ionic liquid, respectively. BCNs prepared from exfoliated graphene show capacitance in the 276-306 $\mathrm{F} \mathrm{g}^{-1}$ range [31,105].

Layered TMDs such as $\mathrm{MoS}_{2}$ and $\mathrm{WS}_{2}$ are being explored in the area for supercapacitor applications [1]. Thus, chemically exfoliated $1 \mathrm{~T} \mathrm{MoS}_{2}$ nanosheets exhibit capacitance values ranging from 400 to $700 \mathrm{~F} \mathrm{~cm}^{-3}$ in aqueous electrolytes and the material can be charged up to a high voltage (3.5 V) of organic electrolytes [106]. Porous $\mathrm{MoS}_{2}$ nanosheets exhibit a capacitance of $330 \mathrm{mF} \mathrm{cm}^{-3}$ with high power density and cyclic stability [107]. Laser-patterned $\mathrm{MoS}_{2}$ nanofilms exhibit a volumetric capacitance of $178 \mathrm{~F} \mathrm{~cm}^{-3}$, which is better than that of some of the graphene-based 

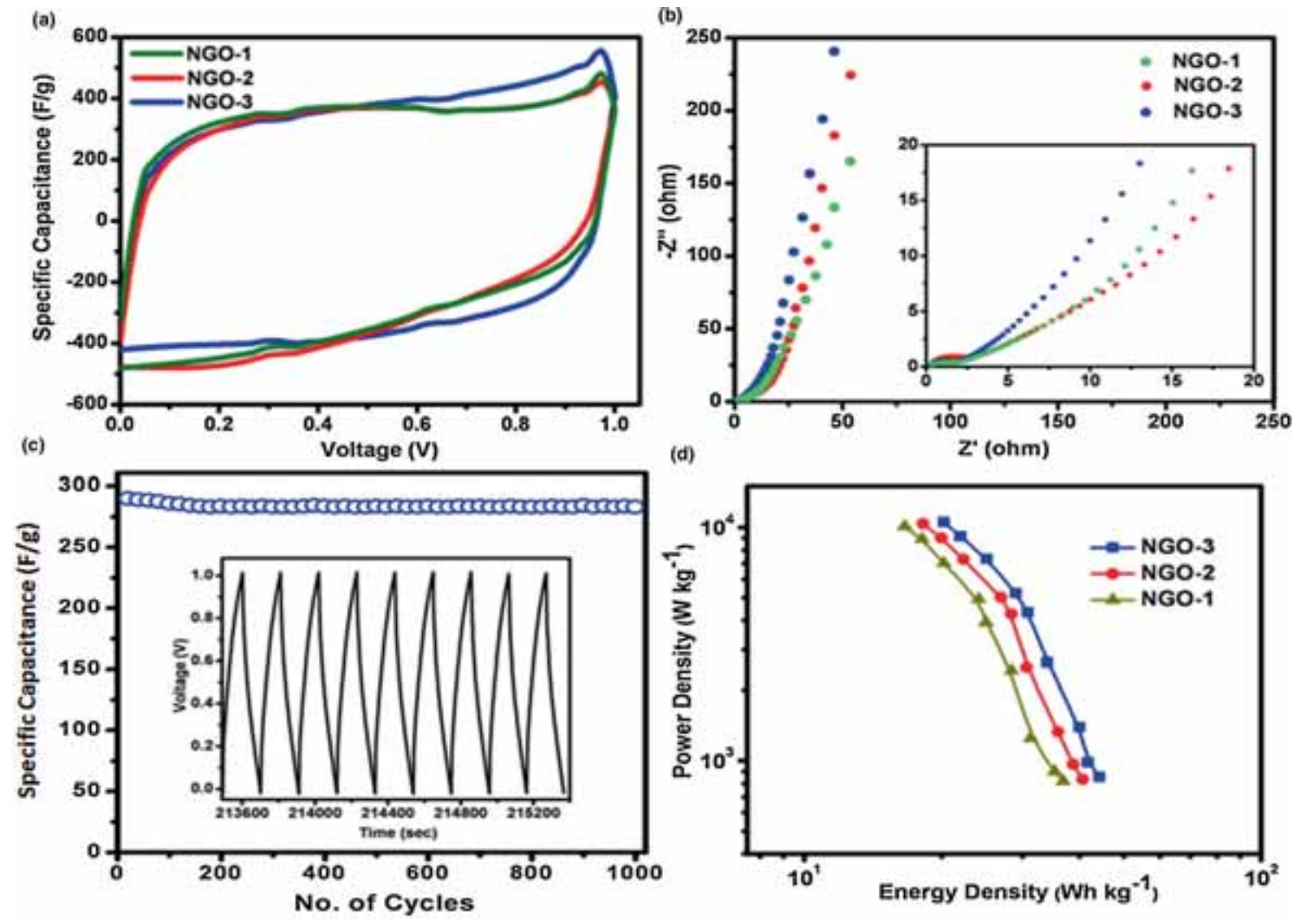

Figure 4. (a) Cyclic voltammograms of NGOs at a scan rate of $20 \mathrm{mV} \mathrm{s}^{-1}$. (b) Nyquist curves for NGO electrodes. (c) Cyclic stability of NGOs (the inset shows the charge-discharge curves of the last few cycles). (d) Ragone plots of NGO-based supercapacitors. Adapted from ref. [97]. Copyright 2013 Royal Society of Chemistry.

devices [108]. The capacitance of $\mathrm{MoS}_{2}$ can be tuned by making composites with graphene, where the $\mathrm{MoS}_{2}$-graphene composites have shown extraordinary supercapacitor properties [109-111].

\section{Oxygen reduction reaction}

The oxygen reduction reaction (ORR) plays an important role in fuel cell technology and the reaction kinetics are generally sluggish. The most used catalyst is $\mathrm{Pt}$, which is expensive and less abundant. 2D materials like graphene and transition metal chalcogenides have shown good prospects as ORR catalysts [1]. Nitrogen-doped graphene shows phenomenal ORR activity with an electron transfer of 4 . Nitrogen-doped graphene synthesized by CVD shows excellent ORR activity in alkaline medium with good stability and overpotential [112,113]. Qu et al [112] have shown that N-doped graphene is resistant towards carbon monoxide poisoning when compared with platinum in alkaline medium. Rotating ring-disk electrode (RRDE) voltammograms of the $\mathrm{N}$-graphene film are shown in figure 5a. Nitrogen-doped graphene shows almost 3 times higher current density compared with that of platinum with an electron transfer ranging from 3.6 to 4 . Electrocatalytic oxidation of methanol is shown in figure $5 \mathrm{~b}$, with $\mathrm{N}$-graphene showing excellent stability. Pt-C electrode gets poisoned under carbon monoxide $(\mathrm{CO})$, whereas $\mathrm{N}$ graphene electrode is unresponsive under the same conditions as shown in figure $5 \mathrm{c}$. $\mathrm{N}$-graphene electrode shows excellent stability towards ORR even after 200000 continuous cycles (figure $5 \mathrm{~d}$ ). $\mathrm{N}$-doped graphene prepared by pyrolysis of polyaniline with graphene shows good ORR activity with large kinetic-current density [114]. N-doped graphene synthesized by ball milling [115], pyrolysis of GO-melamine and GO-dicyandiamide [116] and microwave [97] are also reported to exhibit good electrocatalytic activity comparable to that of platinum. Theoretical calculations predict that nitrogen doping in graphene introduces asymmetry spin density and atomic charge density, which in turn enhances the electrocatalytic activity [117].

High-surface-area BCNs with different proportions of nitrogen and boron show excellent ORR activity with an electron transfer of 4 in alkaline medium [118], whereas, BCNs made with thermal annealing of GO with boric acid and ammonia have shown good ORR activity and methanol tolerance better than that of platinum [119]. BCNs made from exfoliated graphene have shown similar electrocatalytic activity $[31,105]$. It is to be noted that boron-doped [120] and $\mathrm{B}, \mathrm{N}$-doped graphene [121] are also reported to show good ORR activity. 

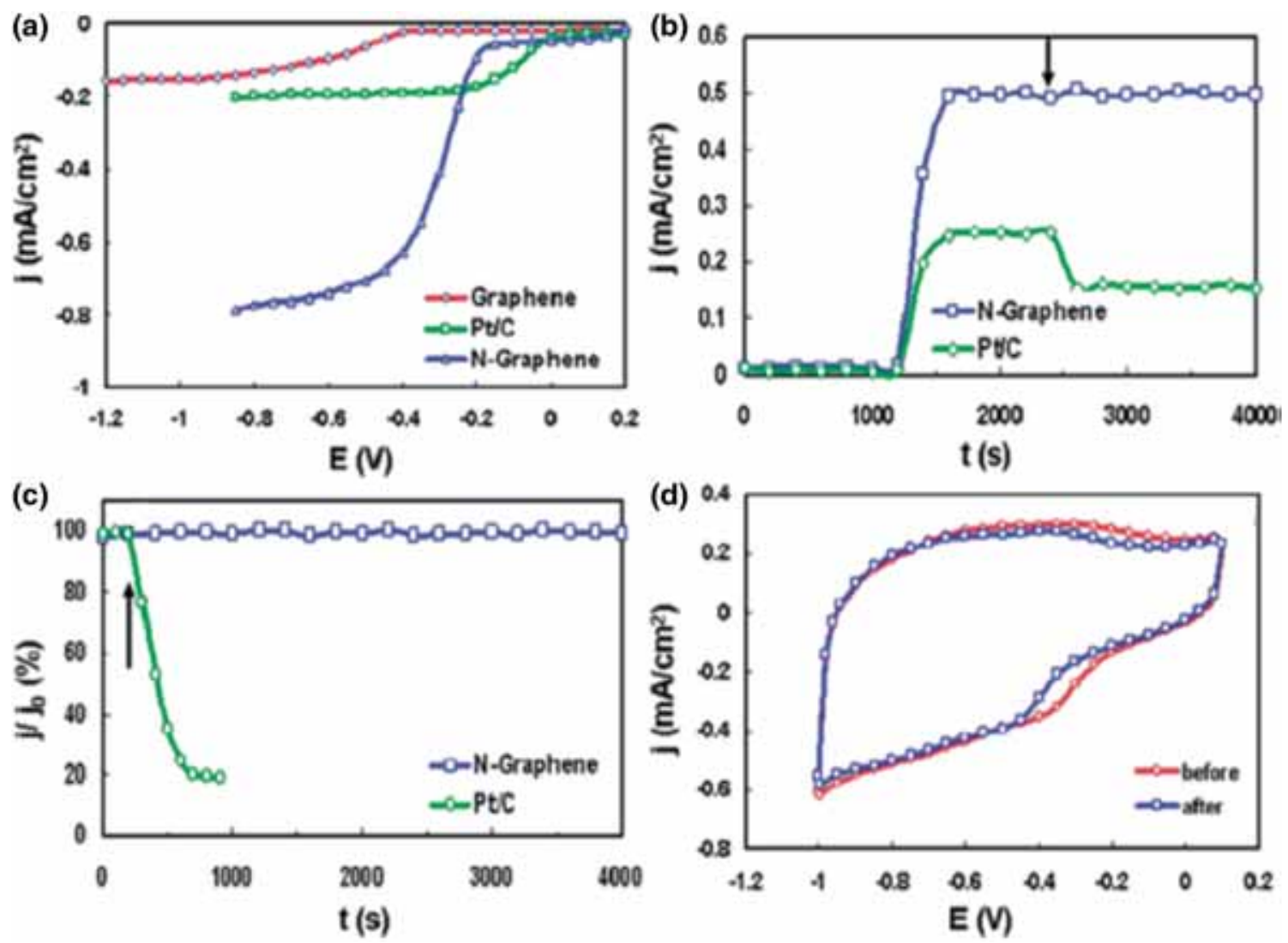

Figure 5. (a) RRDE voltammograms for the ORR in air-saturated $0.1 \mathrm{M} \mathrm{KOH}$. (b) Current density $(j)-$ time $(t)$ chronoamperometric responses obtained at the Pt-C. (c) Current $(j)$-time $(t)$ chronoamperometric response of Pt-C and $\mathrm{N}$-graphene electrodes to $\mathrm{CO}$. (d) Cyclic voltammograms of $\mathrm{N}$-graphene electrode a continuous potentiodynamic swept for 200000 cycles. Adapted from ref. [112]. Copyright 2010 American Chemical Society.

$\mathrm{MoS}_{2}$ particles show improved ORR catalytic activity compared with bulk $\mathrm{MoS}_{2}$ [122]. The size and the exposed Mo edges of the $\mathrm{MoS}_{2}$ play a role in the enhanced activity. $\mathrm{MoS}_{2}$-graphene nanocomposites have shown significant ORR activity compared with pristine $\mathrm{MoS}_{2}$ [110]. $\mathrm{MoS}_{2}$ composites with several carbon materials are also reported to exhibit better activity when compared with $\mathrm{MoS}_{2}$ [123].

\section{Lithium-ion batteries}

Lithium-ion batteries are playing increasingly important role in electronic devices and in energy storage due to their high energy density and power density capabilities. 2D materials like graphene and $\mathrm{MoS}_{2}$ show good charge storing ability because of the fast ion-conduction and reversible Li-intercalation and de-intercalation properties [5]. Nitrogendoped graphene deposited on $\mathrm{Cu}$ foils have shown double reversible discharge capacity [124]. Thermally prepared nitrogen-doped graphene from GO and ammonia exhibits a reversible capacity of $684 \mathrm{mAh} \mathrm{g}^{-1}$. Glucose-based synthesis of nitrogen-doped graphene with different nitrogen dopants enhanced the reversible capacity of $832.4 \mathrm{mAh} \mathrm{g}^{-1}$ with very good cycling stability [125]. BCNs prepared by hightemperature reaction of carbon, boric acid and urea have shown exceptional stability of more than 100 cycles and exhibit specific capacities ranging from 710 to $150 \mathrm{mAh} \mathrm{g}^{-1}$ [126].

Haering et al [127] reported the first $\mathrm{MoS}_{2}$-based Li-batteries with a charge capacity of $750 \mathrm{mAh} \mathrm{g}^{-1}$ after 20 cycles at a current density of $50 \mathrm{~mA} \mathrm{~g}^{-1}$. The charge capacity can be increased up to $\sim 1000 \mathrm{mAh} \mathrm{g}^{-1}$ by making the $\mathrm{MoS}_{2}$ nanosheets hydrothermally [128]. In addition to $\mathrm{Li}$ ion batteries, $\mathrm{MoS}_{2}$ nanowalls grown on alumina substrates using atomic layer deposition exhibit exceptional sodium ion battery capabilities [129]. The nanowalls show a specific capacity value of 2726 and $2078 \mathrm{mAh} \mathrm{g}^{-1}$ in the 1st and 10th cycle, respectively (figure 6a). Stable capacity is observed in the voltage range of 1.2-3.0 V, yielding a reversible capacity of 980 and $590 \mathrm{mAh} \mathrm{g}^{-1}$ in 1st and 100th cycles, respectively (figure $6 \mathrm{~b}$ and $\mathrm{c}$ ). The capacities were stable even at high current densities (figure 6d). $\mathrm{MoS}_{2}$-graphene improves the reversible capacity of $1290 \mathrm{mAh} \mathrm{g}^{-1}$ at a current density of $100 \mathrm{~mA} \mathrm{~g}^{-1}$ up to 50 cycles [130]. 

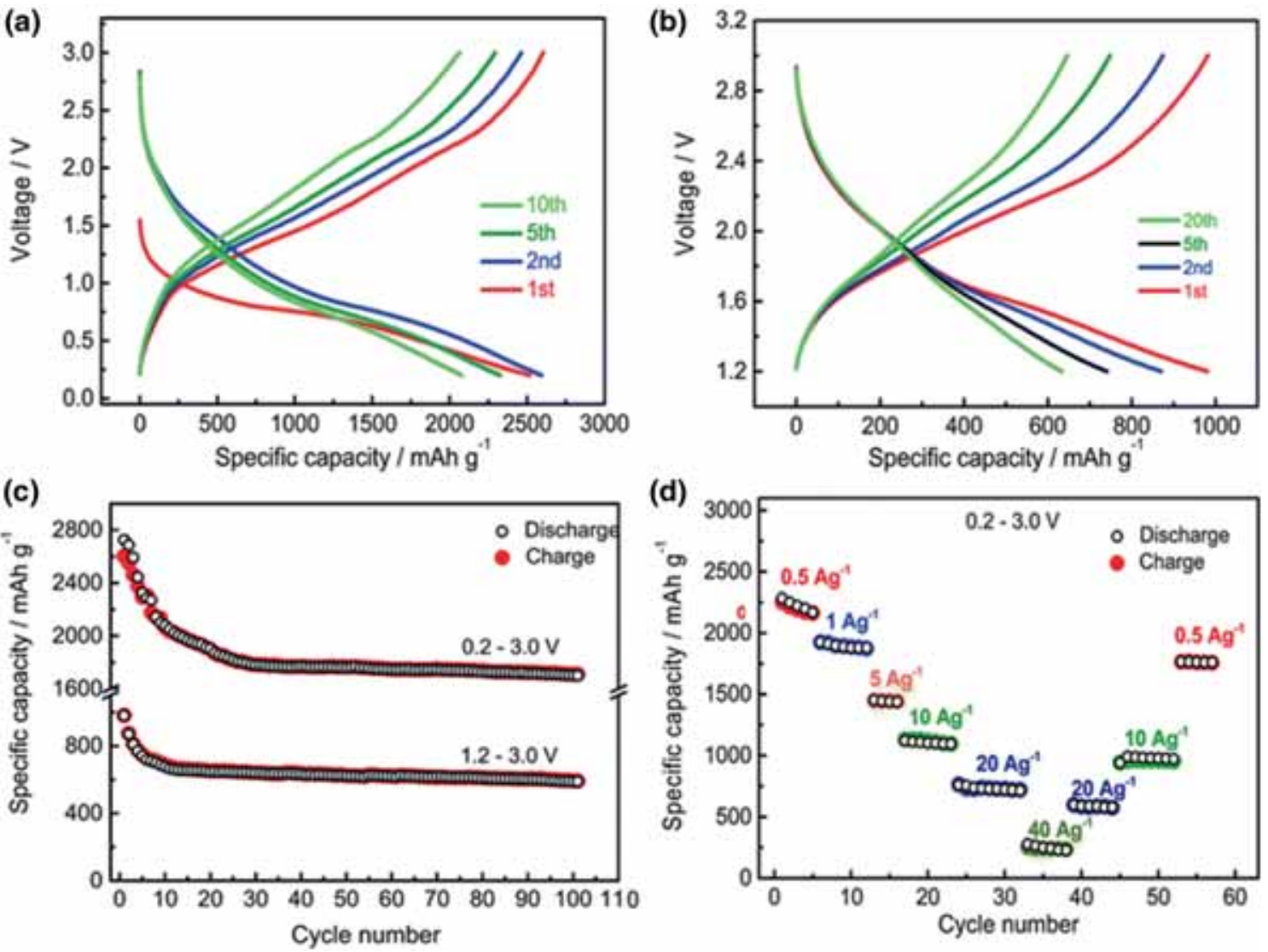

Figure 6. (a, b) Galvanostatic charge-discharge curves in different voltage ranges, (c) cycling stability at $0.5 \mathrm{~A} \mathrm{~g}^{-1}$ and (d) rate capability of ALD-grown $\mathrm{MoS}_{2}$ vs. Li for 0.2-3.0 V. Adapted from ref. [129]. Copyright 2018 Royal Society of Chemistry.

\section{Hydrogen evolution reaction}

Hydrogen evolution reaction (HER) refers to splitting water into hydrogen usually in the presence of sacrificial reagents to enhance the reaction by overcoming the slow water oxidation by a facile one. HER can be achieved either by electrochemical, photochemical or photo-electrochemical means and photochemical method is being used preferably. In electrochemical HER, a catalyst with thermo-neutral onset potential $(\Delta G \sim 0)$ catalyst like $\mathrm{Pt}$ is being used. However, the scalability is limited due to the exorbitant cost of Pt. Two-dimensional TMDs have shown promising HER activity with near-thermoneutral onset potential. Moreover, these materials offer strong structure and activity relationship, which offers tremendous scope to improve the activity of these catalysts through various strategies.

Graphene-based catalysts are stable and durable with good electrical and thermal properties beneficial for the conduction of heat generated during reactions, the good electric conductivity rendering the materials candidates as electrocatalysts and as supports. Introduction of edges, defects, dislocations or doping with non-metals favours the catalytic reactions
[131]. Functionalizing with oxygen, hydrogen or halogens can also alter the electronic properties of graphene. BCNs, especially $\mathrm{BC}_{7} \mathrm{~N}$, have shown high potential to replace $\mathrm{Pt}$, in terms of low onset-potential, high current density along with commercial scalability as they are a non-metal catalyst [132]. GO possesses a band gap that depends on the degree of functionalization and exhibits HER in pure water and in aqueous methanol solution on irradiation [133], which is further enhanced by loading $\mathrm{Ni}$ or $\mathrm{NiO}$ [134]. However, GO is primarily used with direct-band-gap semiconductors like $\mathrm{TiO}_{2}, \mathrm{CdS}$ or dye (Eosin $\mathrm{Y}$ ) as an electron transport bridge or redox mediator due to its high electronic conductivity for HER [135].

Recently, $\mathrm{MoS}_{2}$ nanostructures have received considerable attention for HER. The edge sites can be increased by vertically growing $\mathrm{MoS}_{2}$ or forming inorganic fullerenes (IFs). Nanostructuring can increase the number of active sites and thereby increase current density. Seo et al [136] find that increasing the interlayer spacing between $\mathrm{MoS}_{2}$ layers increases the HER activity of $\mathrm{MoS}_{2}$ colloids significantly (figure 7a). The increased inter-layer spacing allows easy access of water molecules to HER site. The basal planes can 

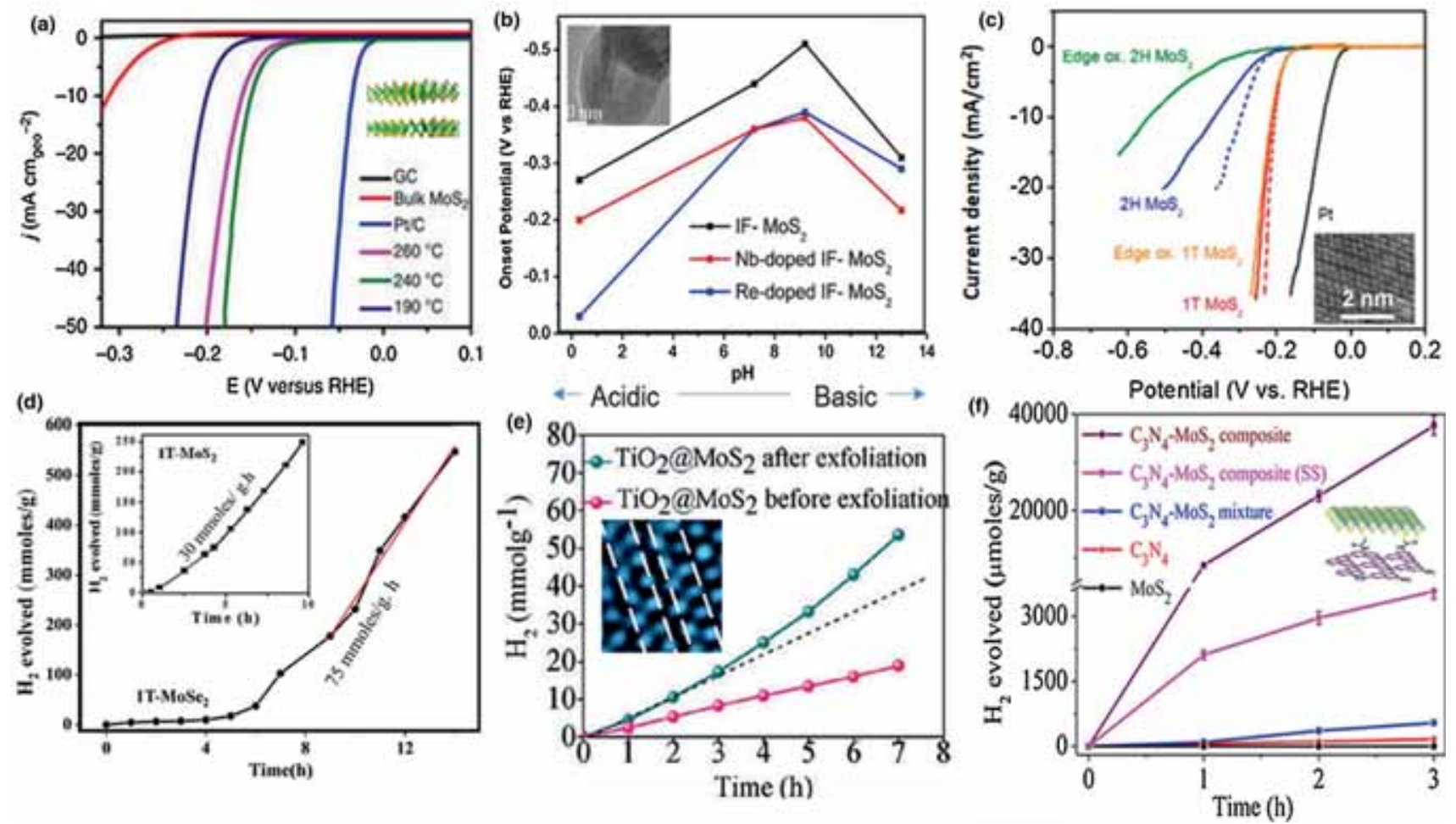

Figure 7. (a) Nanostructuring of $\mathrm{MoS}_{2}$ increases the interlayer spacing between the layers, allowing water molecules, along with creation of edge sites; edge sites along with defect sites increase the HER activity of the catalyst. The activity of nanostructured MoS 2 is higher than that of bulk $\mathrm{MoS}_{2}$. Adapted from ref. [136]. (b) Doped-inorganic fullerene (IF)-MoS 2 (inset) integrates the idea of activating basal planes by doping in addition to maximizing edge sites by morphology change. The doped sample is stable and works at all $\mathrm{pH}$ levels. Adapted from ref. [138]. (c) The phase-engineered 1T-form of $\mathrm{MoS}_{2}$ shows high current density compared with $2 \mathrm{H}-\mathrm{MoS} 2$, which is attributed to current-active basal planes. The oxidized edge sites of 1T-form show high HER activity, which implies that the basal plane is also active unlike in 2H-form. Adapted from ref. [139]. (d) Similarly, 1T-form of $\mathrm{MoS}_{2}$ and $\mathrm{MoSe}_{2}$ shows high HER activity in Eosin Y (EY) dye-sensitized in triethanolamine solution. Adapted from ref. [142]. (e) $1 \mathrm{~T}-\mathrm{MoS}_{2}$ was stabilized on $\mathrm{TiO}_{2}$, which showed stability for a year (inset image). The 1T-form is self-stabilized during HER evolution in dye-based HER. Adapted from ref. [143]. (f) The covalently linked $\mathrm{C}_{3} \mathrm{~N}_{4}-\mathrm{MoS}_{2}$ is highly active as compared with its individual components or physical mixture. The covalently linked sample has greater charge transfer as compared with physical mixture. Adapted from ref. [26]. Copyright 2013, 2015 and 2017 American Chemical Society. Copyright 2013 and 2016 John Wiley and Sons Ltd. Copyright 2014 AIP Publishing.

be activated either by creating a vacancy, phase-engineering, doping or substitution with other transition metals $(\mathrm{Nb}, \mathrm{Re})$ or non-metals ( $\mathrm{Se}, \mathrm{Cl}$ or $\mathrm{P})$ [137]. Chhetri et al [138] have integrated two strategies simultaneously by the modifying electronic structure coupled with increased edge sites in a doped IF of $\mathrm{MoS}_{2}$. Re- and Nb-doped in IF-MoS 2 with lower onset potential exhibit increased HER activity. The catalysts perform at all $\mathrm{pH}$ values $(0-14)$ and even in sea-water (figure 7b) [138]. Another strategy to activate both basal planes and edge sites is by phase engineering, by transforming the stable $2 \mathrm{H}-\mathrm{MoS}_{2}$ to metallic-1T-MoS 2 , which activates the basal planes along with increasing the overall conductivity of the film [139]. The edge sites of 1T-form did not show any decrease in activity, whereas the oxidized edgesite $2 \mathrm{H}-\mathrm{MoS}_{2}$ have shown a considerable decrease in activity. The study signifies the importance of active basal planes in 1T-form for its enhanced activity (figure 7c). Covalently linked $\mathrm{MoS}_{2}$ with $\mathrm{BCN}$ have shown high activity with a low-onset potential close to Pt [25]. Hybrid composites of TMDs with other materials such as carbonaceous materials or metals can enhance the efficiency synergistically [140].

In photochemical HER, $\mathrm{MoS}_{2}$ has shown immense potential as the catalyst owing to its suitable band position. However, due to indirect band gap, it needs an external lightharvesting system like a dye or semiconductor to harvest its full potential [137]. $2 \mathrm{H}-\mathrm{MoS}_{2}$ has poor catalytic HER performance and needs to be modified. Rao and co-workers have demonstrated superior catalytic activity of the metallic 1Tphase over that of the semiconducting 2H-phase in $\mathrm{MoSe}_{2}$ and $\mathrm{MoS}_{2}$. In the Eosin Y(EY)-triethanolamine (TEOA) based photosystem, 1T-phase exhibits an activity 600 and 900 times superior to that of $2 \mathrm{H}$-phase for $\mathrm{MoS}_{2}$ and $\mathrm{MoS}_{2}$, respectively. As the HER activity of the basal planes is similar to that of edge sites, 1T-phase has superior activity. The strategy captures the idea of nanostructuring, activating 


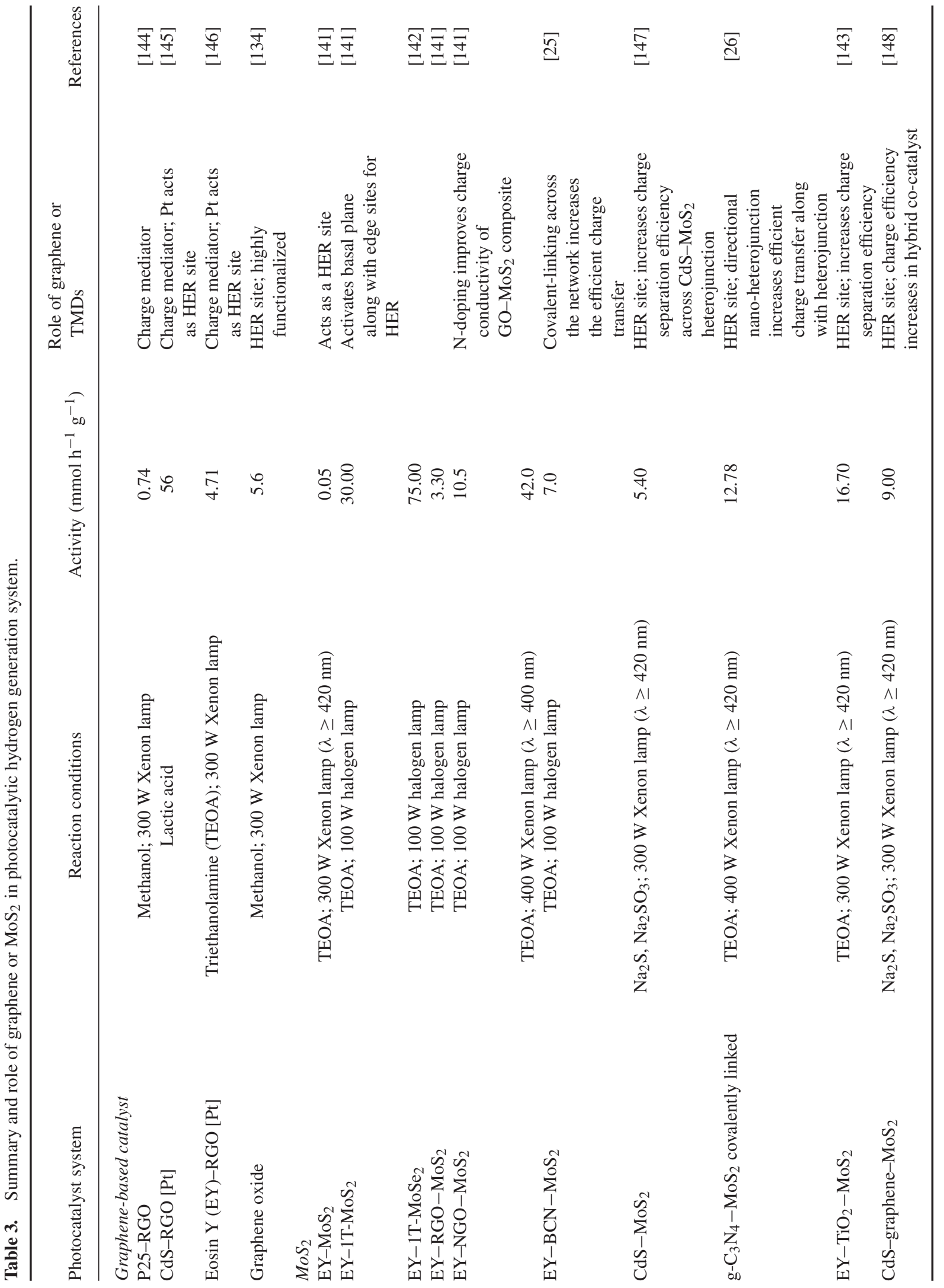


basal planes coupled with increased metallicity for better charge transport in and between the layers (figure 7d) $[141,142]$. However, the 1T-phase is less stable, and therefore the remarkable HER activity cannot be entirely harvested. Duan and co-workers have grown $\mathrm{TiO}_{2}$ nanotubes and grafted $1 \mathrm{~T}-\mathrm{MoS}_{2}$ on its surface using an Eosin $\mathrm{Y}$ dye-sensitized system where the $1 \mathrm{~T}-\mathrm{MoS}_{2}$ phase stabilizes itself during the process of hydrogen evolution and it is stable even after one year. The adsorption on $\mathrm{H}$ atoms along with the catalyst support stabilizes the phase, which can provide robust, high-performing catalyst for long-term use (figure 7e) [143]. Covalently linking 2H$\mathrm{MoS}_{2}$ to other 2D materials like $\mathrm{BCN}$ and $\mathrm{C}_{3} \mathrm{~N}_{4}$ is a way to solve most of the problems by integrating multiple problems $[25,26]$. The layer-by-layer directional linking of $\mathrm{C}_{3} \mathrm{~N}_{4}$ with $\mathrm{MoS}_{2}$ increases the activity of $\mathrm{C}_{3} \mathrm{~N}_{4}$ by 246 fold. The enhanced activity is attributed to charge transfer across the network through space and bonds because of the direct overlapping of the layers and short conducting bonds (figure 7f) [26]. Table 3 summarizes the role of graphene and TMDs in photocatalytic hydrogen generation.

\section{Mechanical properties}

Graphene possesses unique mechanical properties with an elastic modulus of $\sim 1 \mathrm{TPa}$ and a theoretical strength of $\sim 130 \mathrm{GPa}$. Graphene can be used as a reinforcement in polymer matrix such as poly(methyl methacrylate) (PMMA) or polyvinyl alcohol (PVA). The mechanical properties of graphene (0.6 wt\%)-reinforced PVA and PMMA-matrix composites show an increase in both the elastic modulus (3.65 GPa) and hardness (153 MPa) [149]. The mechanical properties of the graphene-polymer matrix were further improved by adding single-walled carbon nanotubes [150]. Figure 8 shows the variation of the nanohardness $(H) v s$. elastic modulus $(E)$ as a function of the nanocarbon content. The synergy between the two nanocarbons increases the mechanical properties up to $400 \%$. Graphene-like boron nitride has shown improved mechanical properties depending upon the number of layers. When three BN layers are incorporated in PMMA matrix, the hardness and elastic modulus increased up to $125 \%$ and $130 \%$, respectively [151]. Theoretical calculations predict that $\mathrm{MoS}_{2}$ is mechanically stable and can withstand extra strains with an inplane stiffness as high as $184 \mathrm{GPa}$ [133]. In the case of graphene- $\mathrm{MoS}_{2}$ - graphene heterostructure the Young's modulus increases almost three times higher than that of single layer $\mathrm{MoS}_{2}$ theoretically [152]. Some recent studies being carried out here have shown that polymer composites of BCNs exhibit synergy in mechanical properties relative to graphene and BN. Furthermore, graphene can be grafted onto MOFs and other materials to improve their mechanical properties [153].

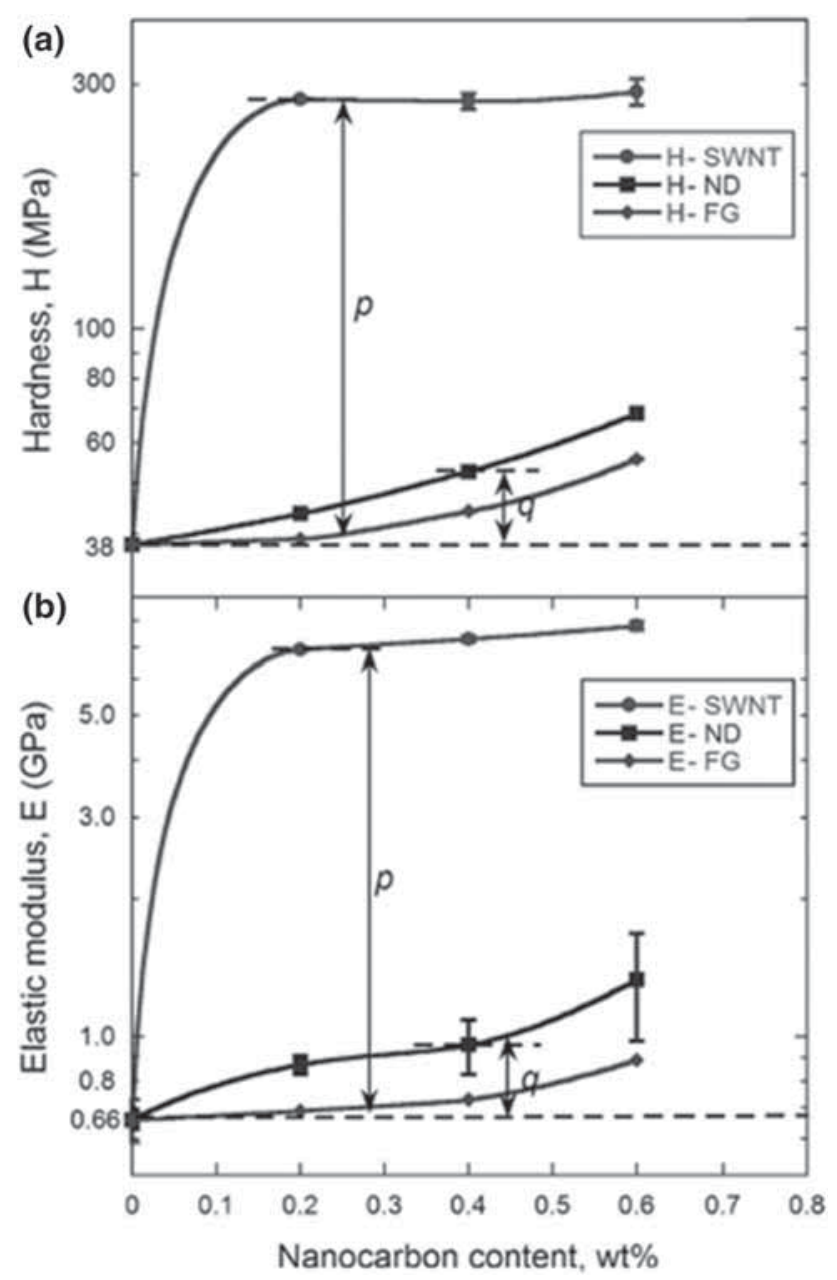

Figure 8. Mechanical properties (a) hardness and (b) elastic modulus with the variation of nanocarbon content. Singlewalled nanotube (SWNT)-reinforced nanocomposites give superior mechanical properties compared with the few-layer graphene (FG) and nanodiamond (ND). Adapted from ref. [150]. Copyright 2009 National Academy of Sciences (USA).

\section{Conclusions}

The previous sections would suffice to indicate the fascinating properties exhibited by graphene, $\mathrm{MoS}_{2}$ and other 2D materials. The subject is being pursued widely, and much more will be done in the next few years. This is particularly because of the properties worthy of practical applications. For example, in the HER, $\mathrm{MoS}_{2^{-}}$and $\mathrm{BCN}$-based materials exhibit excellent activity. Similarly, $\mathrm{MoS}_{2}$ is useful in fabricating Na-ion batteries with desirable characteristics. We should not forget that graphene itself has found applications in supercapacitors and other areas related to electronic and energy devices. Being a direct band gap material, $\mathrm{MoS}_{2}$ exhibits unique properties such as intense photoluminescence and circular luminescence. Photoluminescence of single-layer $\mathrm{MoS}_{2}$ can be used for sensor applications. Optical and electronic properties of $\mathrm{MoS}_{2}$ and other 2D materials are 
indeed very attractive. There are likely to be applications of heterojunctions, covalently cross-linked structures as well as van der Waals structures.

\section{References}

[1] Rao C N R, Gopalakrishnan K and Maitra U 2015 ACS Appl. Mater. Interfaces 77809

[2] Rao C N R and Waghmare U V 2017 2D inorganic materials beyond graphene $476 \mathrm{pp}$

[3] Rao C N R, Gopalakrishnan K and Govindaraj A 2014 Nano Today 9324

[4] Rao C N R and Gopalakrishnan K 2017 ACS Appl. Mater. Interfaces 919478

[5] Rao C N R, Maitra U and Waghmare U V 2014 Chem. Phys. Lett. 609172

[6] Rao C N R, Pramoda K and Kumar R 2017 Chem. Commun. 5310093

[7] Rao C N R and Sood A K 2013 Graphene: synthesis, properties, and phenomena (Weinheim, Germany: Wiley-VCH)

[8] Novoselov K S, Jiang D, Schedin F, Booth T J, Khotkevich V V, Morozov S V et al 2005 Proc. Natl. Acad. Sci. USA 102 10451

[9] Rao C N R, Sood A K, Subrahmanyam K S and Govindaraj A 2009 Angew. Chem. Int. Ed. 487752

[10] Subrahmanyam K S, Panchakarla L S, Govindaraj A and Rao C N R 2009 J. Phys. Chem. C 1134257

[11] Panchakarla L S, Subrahmanyam K S, Saha S K, Govindaraj A, Krishnamurthy H R, Waghmare U V et al 2009 Adv. Mater. 214726

[12] Wei D, Liu Y, Wang Y, Zhang H, Huang L and Yu G 2009 Nano Lett. 91752

[13] Li X, Wang H, Robinson J T, Sanchez H, Diankov G and Dai H 2009 J. Am. Chem. Soc. 13115939

[14] Kumar N, Moses K, Pramoda K, Shirodkar S N, Mishra A K, Waghmare U V et al 2013 J. Mater. Chem. A 15806

[15] Smith R J, King P J, Lotya M, Wirtz C, Khan U, De S et al 2011 Adv. Mater. 233944

[16] Coleman J N, Lotya M, O’Neill A, Bergin S D, King P J, Khan U et al 2011 Science 331568

[17] Joensen P, Frindt R F and Morrison S R 1986 Mater. Res. Bull. 21457

[18] Eda G, Yamaguchi H, Voiry D, Fujita T, Chen M and Chhowalla M 2011 Nano Lett. 115111

[19] Wang S, Rong Y, Fan Y, Pacios M, Bhaskaran H, He K et al 2014 Chem. Mater. 266371

[20] Shi J, Zhang X, Ma D, Zhu J, Zhang Y, Guo Z et al 2015 ACS Nano 94017

[21] Feng Q, Zhu Y, Hong J, Zhang M, Duan W, Mao N et al 2014 Adv. Mater. 262648

[22] Liu K K, Zhang W, Lee Y-H, Lin Y-C, Chang M-T, Su C-Y et al 2012 Nano Lett. 121538

[23] Gao M-R, Xu Y-F, Jiang J and Yu S-H 2013 Chem. Soc. Rev. 422986

[24] Mahler B, Hoepfner V, Liao K and Ozin G A 2014 J. Am. Chem. Soc. 13614121

[25] Pramoda K, Ayyub M M, Singh N K, Chhetri M, Gupta U, Soni A et al 2017 J. Phys. Chem. C https://doi.org/10.1021/ acs.jpcc.7b10782
[26] Pramoda K, Gupta U, Chhetri M, Bandyopadhyay A, Pati S K and Rao C N R 2017 ACS Appl. Mater. Interfaces 9 10664

[27] Castro Neto A H, Guinea F, Peres N M R, Novoselov K S and Geim A K 2009 Rev. Modern Phys. 81109

[28] Fang T, Konar A, Xing H and Jena D 2008 Phys. Rev. B 78 205403

[29] Daeha J, Chunder A, Lei Z and Saiful I K 2010 Nanotechnology 21165202

[30] Fiori G, Betti A, Bruzzone S and Iannaccone G 2012 ACS Nano 62642

[31] Sreedhara M B, Gopalakrishnan K, Bharath B, Kumar R, Kulkarni G U and Rao C N R 2016 Chem. Phys. Lett. 657 124

[32] Jena D 2013 Proc. IEEE 1011585

[33] Schwierz F, Pezoldt J and Granzner R 2015 Nanoscale 78261

[34] Chhowalla M, Shin H S, Eda G, Li L-J, Loh K P and Zhang H 2013 Nat. Chem. 5263

[35] Ayari A, Cobas E, Ogundadegbe O and Fuhrer M S $2007 \mathrm{~J}$. Appl. Phys. 101014507

[36] Late D J, Liu B, Matte H S S R, Dravid V P and Rao C N R 2012 ACS Nano 65635

[37] Radisavljevic B, Radenovic A, Brivio J, Giacometti V and Kis A 2011 Nat. Nanotechnol. 6147

[38] Chen F, Xia J, Ferry D K and Tao N 2009 Nano Lett. 92571

[39] Konar A, Fang T and Jena D 2010 Phys. Rev. B 82115452

[40] Jena D and Konar A 2007 Phys. Rev. Lett. 98136805

[41] Yoon Y, Ganapathi K and Salahuddin S 2011 Nano Lett. 11 3768

[42] Radisavljevic B, Whitwick M B and Kis A 2011 ACS Nano 59934

[43] Zhang Y, Ye J, Matsuhashi Y and Iwasa Y 2012 Nano Lett. 121136

[44] Britnell L, Gorbachev R V, Jalil R, Belle B D, Schedin F, Mishchenko A et al 2012 Science 335947

[45] Sarkar D, Xie X, Liu W, Cao W, Kang J, Gong Y et al 2015 Nature $\mathbf{5 2 6} 91$

[46] Novoselov K S, Geim A K, Morozov S V, Jiang D, Zhang Y, Dubonos S V et al 2004 Science 306666

[47] Yuan W and Shi G 2013 J. Mater. Chem. A 110078

[48] Wehling T O, Katsnelson M I and Lichtenstein A I 2009 Chem. Phys. Lett. 476125

[49] Schedin F, Geim A K, Morozov S V, Hill E W, Blake P, Katsnelson M I et al 2007 Nat. Mater. 6652

[50] Hwang S, Lim J, Park H G, Kim W K, Kim D H, Song I S et al 2012 Curr. Appl. Phys. 121017

[51] Yoon H J, Jun D H, Yang J H, Zhou Z, Yang S S and Cheng M M C 2011 Sens. Actuators B: Chem. 157310

[52] Rumyantsev S, Liu G, Shur M S, Potyrailo R A and Balandin A A 2012 Nano Lett. 122294

[53] Berdan R, Prodromakis T, Salaoru I, Khiat A and Toumazou C 2012 App. Phys. Lett. 101243502

[54] Dua V, Surwade S P, Ammu S, Agnihotra S R, Jain S, Roberts $\mathrm{K}$ E et al 2010 Angew. Chem. Int. Ed. 492154

[55] Bi H, Yin K, Xie X, Ji J, Wan S, Sun L et al 2013 Sci. Rep. 3 2714

[56] Yuan W, Liu A, Huang L, Li C and Shi G 2013 Adv. Mater. 25766

[57] Shao Y, Wang J, Wu H, Liu J, Aksay I A and Lin Y 2010 Electroanalysis 221027

[58] Zhou M, Zhai Y and Dong S 2009 Anal. Chem. 815603 
[59] He Q, Sudibya H G, Yin Z, Wu S, Li H, Boey F et al 2010 ACS Nano 43201

[60] Sudibya H G, He Q, Zhang H and Chen P 2011 ACS Nano 5 1990

[61] Dubovik T V, Itsenko A I, Dolinskaya L P, Zyatkevich N S and Levkov L Y 1995 Powder Met. Met. Ceram. 33 100

[62] Pramoda K, Moses K, Maitra U and Rao C N R 2015 Electroanalysis 271892

[63] Li H, Wu J, Yin Z and Zhang H 2014 Acc. Chem. Res. 47 1067

[64] Late D J, Huang Y-K, Liu B, Acharya J, Shirodkar S N, Luo J et al 2013 ACS Nano 74879

[65] Zhu C, Zeng Z, Li H, Li F, Fan C and Zhang H 2013 J. Am. Chem. Soc. 1355998

[66] Zhang Y, Zheng B, Zhu C, Zhang X, Tan C, Li H et al 2015 Adv. Mater. 27935

[67] Graf M, Liu K, Sarathy A, Leburton J P and Radenovic A 2017 Biophys. J. 114180

[68] Lim S, Kang B, Kwak D, Lee W H, Lim J A and Cho K 2012 J. Phys. Chem. C 1167520

[69] Perkins F K, Friedman A L, Cobas E, Campbell P M, Jernigan G G and Jonker B T 2013 Nano Lett. 13668

[70] Nair R R, Blake P, Grigorenko A N, Novoselov K S, Booth T J, Stauber T et al 2008 Science 3201308

[71] Wang F, Zhang Y, Tian C, Girit C, Zettl A, Crommie M et al 2008 Science 320206

[72] Chitara B, Panchakarla L S, Krupanidhi S B and Rao C N R 2011 Adv. Mater. 235419

[73] Chitara B, Krupanidhi S B and Rao C N R 2011 App. Phys. Lett. 99113114

[74] Lee H S, Min S-W, Chang Y-G, Park M K, Nam T, Kim H et al 2012 Nano Lett. 123695

[75] Rao C N R, Matte H S S R and Subrahmanyam K S 2013 Acc. Chem. Res. 46149

[76] Chou S S, Kaehr B, Kim J, Foley B M, De M, Hopkins P E et al 2013 Angew. Chem. Int. Ed. $\mathbf{5 2} 4160$

[77] Yin Z, Li H, Li H, Jiang L, Shi Y, Sun Y et al 2012 ACS Nano 674

[78] Lopez-Sanchez O, Lembke D, Kayci M, Radenovic A and Kis A 2013 Nat. Nanotechnol. 8497

[79] Jariwala D, Marks T J and Hersam M C 2016 Nat. Mater. 16 170

[80] Freitag M, Low T, Xia F and Avouris P 2012 Nat. Photon. 7 53

[81] Wang X, Cheng Z, Xu K, Tsang H K and Xu J-B 2013 Nat. Photon. 7888

[82] Yan J, Kim M H, Elle J A, Sushkov A B, Jenkins G S, Milchberg H M et al 2012 Nat. Nanotechnol. 7472

[83] Britnell L, Ribeiro R M, Eckmann A, Jalil R, Belle B D, Mishchenko A et al 2013 Science 3401311

[84] Baugher B W H, Churchill H O H, Yang Y and Jarillo-Herrero P 2014 Nat. Nanotechnol. 9262

[85] Lee C-H, Lee G-H, van der Zande A M, Chen W, Li Y, Han M et al 2014 Nat. Nanotechnol. 9676

[86] Kotz R and Carlen M 2000 Electrochim. Acta 452483

[87] Zhang L L and Zhao X S 2009 Chem. Soc. Rev. 382520

[88] Vivekchand S R C, Rout C S, Subrahmanyam K S, Govindaraj A and Rao C N R 2008 J. Chem. Sci. 1209

[89] Stoller M D, Park S, Zhu Y, An J and Ruoff R S 2008 Nano Lett. 83498
[90] Kim T, Jung G, Yoo S, Suh K S and Ruoff R S 2013 ACS Nano 76899

[91] Zhu Y, Murali S, Stoller M D, Ganesh K J, Cai W, Ferreira $\mathrm{P}$ J et al 2011 Science 3321537

[92] Zhu Y, Murali S, Stoller M D, Velamakanni A, Piner R D and Ruoff R S 2010 Carbon 482118

[93] Yoo J J, Balakrishnan K, Huang J, Meunier V, Sumpter B G, Srivastava A et al 2011 Nano Lett. 111423

[94] Jeong H M, Lee J W, Shin W H, Choi Y J, Shin H J, Kang J K et al 2011 Nano Lett. 112472

[95] Wen Z, Wang X, Mao S, Bo Z, Kim H, Cui S et al 2012 Adv. Mater. 245610

[96] Gopalakrishnan K, Moses K, Govindaraj A and Rao C N R 2013 Solid State Commun. 17543

[97] Gopalakrishnan K, Govindaraj A and Rao C N R 2013 J. Mater. Chem. A 17563

[98] Sun L, Wang L, Tian C, Tan T, Xie Y, Shi K et al 2012 RSC Adv. 24498

[99] Niu L, Li Z, Hong W, Sun J, Wang Z, Ma L et al 2013 Electrochim. Acta 108666

[100] Zuo Z, Jiang Z and Manthiram A 2013 J. Mater. Chem. A 1 13476

[101] Han J, Zhang L L, Lee S, Oh J, Lee K-S, Potts J R et al 2013 ACS Nano 719

[102] Peng Z, Ye R, Mann J A, Zakhidov D, Li Y, Smalley P R et al 2015 ACS Nano 95868

[103] Li S, Wang Z, Jiang H, Zhang L, Ren J, Zheng M et al 2016 Chem. Commun. 5210988

[104] Thirumal V, Pandurangan A, Jayavel R and Ilangovan R 2016 Synth. Met. 220524

[105] Barua M, Sreedhara M B, Pramoda K and Rao C N R 2017 Chem. Phys. Lett. 683459

[106] Acerce M, Voiry D and Chhowalla M 2015 Nat. Nanotechnol. 10313

[107] Choudhary N, Patel M, Ho Y-H, Dahotre N B, Lee W, Hwang J Y et al 2015 J. Mater. Chem. A 324049

[108] Cao L, Yang S, Gao W, Liu Z, Gong Y, Ma L et al 2013 Small 92905

[109] da Silveira Firmiano E G, Rabelo A C, Dalmaschio C J, Pinheiro A N, Pereira E C, Schreiner W H et al 2014 Adv. Energy Mater. 41301380

[110] Gopalakrishnan K, Pramoda K, Maitra U, Mahima U, Shah M A and Rao C N R 2015 Nanomater. Energy 49

[111] Bissett M A, Kinloch I A and Dryfe R A W 2015 ACS Appl. Mater. Interfaces 717388

[112] Qu L, Liu Y, Baek J-B and Dai L 2010 ACS Nano 41321

[113] Shao Y, Zhang S, Engelhard M H, Li G, Shao G, Wang Y et al 2010 J. Mater. Chem. 207491

[114] Liu M, Song Y, He S, Tjiu W W, Pan J, Xia Y Y et al 2014 ACS Appl. Mater. Interfaces 64214

[115] Fu X, Jin J, Liu Y, Wei Z, Pan F and Zhang J 2014 ACS Appl. Mater. Interfaces 63930

[116] Vikkisk M, Kruusenberg I, Joost U, Shulga E, Kink I and Tammeveski K Appl. Catal. B: Environ. 147369

[117] Zhang L and Xia Z 2011 J. Phys. Chem. C 11511170

[118] Moses K, Kiran V, Sampath S and Rao C N R 2014 Chem. Asian J. 9838

[119] Wang S, Zhang L, Xia Z, Roy A, Chang D W, Baek J-B et al 2012 Angew. Chem. Int. Ed. 514209

[120] Sheng Z-H, Gao H-L, Bao W-J, Wang F-B and Xia X-H 2012 J. Mater. Chem. 22390 
[121] Xue Y, Yu D, Dai L, Wang R, Li D, Roy A et al 2013 Phys. Chem. Chem. Phys. 1512220

[122] Wang T, Gao D, Zhuo J, Zhu Z, Papakonstantinou P, Li Y et al 2013 Chem. Eur. J. 1911939

[123] Rowley-Neale S J, Fearn J M, Brownson D A C, Smith G C, Ji X and Banks C E 2016 Nanoscale 814767

[124] Reddy A L M, Srivastava A, Gowda S R, Gullapalli H, Dubey M and Ajayan P M 2010 ACS Nano 46337

[125] Tian L-L, Wei X-Y, Zhuang Q-C, Jiang C-H, Wu C, Ma G-Y et al 2014 Nanoscale 66075

[126] Sen S, Moses K, Bhattacharyya A J and Rao C N R 2014 Chem. Asian J. 9100

[127] Haering R R et al 1980 US Patent 1804224390

[128] Feng C, Ma J, Li H, Zeng R, Guo Z and Liu H 2009 Mater. Res. Bull. 441811

[129] Sreedhara M B, Gope S, Vishal B, Datta R, Bhattacharyya A J and Rao C N R 2018 J. Mater. Chem. A 62302

[130] Chang K and Chen W 2011 Chem. Commun. 474252

[131] Deng D, Novoselov K S, Fu Q, Zheng N, Tian Z and Bao X 2016 Nat. Nanotechnol. 11218

[132] Chhetri M, Maitra S, Chakraborty H, Waghmare U V and Rao C N R 2016 Energy Environ. Sci. 995

[133] Peng Q and De S 2013 Phys. Chem. Chem. Phys. 1519427

[134] Yeh T-F, Syu J-M, Cheng C, Chang T-H and Teng H 2010 Adv. Funct. Mater. 202255

[135] Yeh T-F, Cihlář J, Chang C-Y, Cheng C and Teng H 2013 Mater. Today 1678

[136] Seo B, Jung G Y, Sa Y J, Jeong H Y, Cheon J Y, Lee J H et al 2015 ACS Nano 93728

[137] Gupta U and Rao C N R 2017 Nano Energy 4149
[138] Chhetri M, Gupta U, Yadgarov L, Rosentsveig R, Tenne R and Rao C N R 2016 ChemElectroChem 31937

[139] Voiry D, Salehi M, Silva R, Fujita T, Chen M, Asefa T et al 2013 Nano Lett. 136222

[140] Lu Q, Yu Y, Ma Q, Chen B and Zhang H 2016 Adv. Mater. 281917

[141] Maitra U, Gupta U, De M, Datta R, Govindaraj A and Rao C N R 2013 Angew. Chem. Int. Ed. 5213057

[142] Gupta U, Naidu B S, Maitra U, Singh A, Shirodkar S N, Waghmare U V et al 2014 APL Mater. 2092802

[143] Wang L, Liu X, Luo J, Duan X, Crittenden J, Liu C et al 2017 Angew. Chem. Int. Ed. 1297718

[144] Fan W, Lai Q, Zhang Q and Wang Y 2011 J. Phys. Chem. C 11510694

[145] Li Q, Guo B, Yu J, Ran J, Zhang B, Yan H et al 2011 J. Am. Chem. Soc. 13310878

[146] Zhang W, Li Y, Peng S and Cai X 2014 Beilstein J. Nanotechnol. 5801

[147] Zong X, Wu G, Yan H, Ma G, Shi J, Wen F et al 2010 J. Phys. Chem. C 1141963

[148] Liu M, Li F, Sun Z, Ma L, Xu L and Wang Y 2014 Chem. Commun. 5011004

[149] Barun D, Prasad K E, Ramamurty U and Rao C N R 2009 Nanotechnology 20125705

[150] Prasad K E, Das B, Maitra U, Ramamurty U and Rao C N R 2009 Proc. Natl. Acad. Sci. USA 10613186

[151] Kiran M S R N, Raidongia K, Ramamurty U and Rao C N R 2011 Scr. Mater. 64592

[152] Jiang J-W and Park H S 2014 Appl. Phys. Lett. 105033108

[153] Kumar R, Raut D, Ramamurty U and Rao C N R 2016 Angew. Chem. Int. Ed. 1287988 